Aus der Klinik für Pädiatrische Kardiologie und Intensivmedizin

(Prof. Dr. med. T. Paul)

der Medizinischen Fakultät der Universität Göttingen

\title{
Auswirkung von drei konsekutiven \\ Kryoenergieapplikationen auf die Bildung und \\ Größe von Ablationsläsionen und die \\ Koronararterien im sich entwickelnden Myokard
}

\author{
INAUGURAL-DISSERTATION \\ zur Erlangung des Doktorgrades \\ der Medizinischen Fakultät der \\ Georg-August-Universität zu Göttingen
}

vorgelegt von

Filipe Abreu da Cunha

aus

Bremen

Göttingen 2019 
Dekan:

Referent/in

Ko-Referent/in:

Drittreferent/in:
Prof. Dr. med. W. Brück

PD Dr. med. U. Krause

Datum der mündlichen Prüfung: 
Hiermit erkläre ich, die Dissertation mit dem Titel "Auswirkung von drei konsekutiven Kryoenergieapplikationen auf die Bildung und Größe von Ablationsläsionen und die Koronararterien im sich entwickelnden Myokard" eigenständig angefertigt und keine anderen als die von mir angegebenen Quellen und Hilfsmittel verwendet zu haben.

Göttingen, den

(Unterschrift) 
Die Daten, auf denen die vorliegende Arbeit basiert, wurden teilweise publiziert:

Krause U, Abreu da Cunha FD, Backhoff D, Jacobshagen C, Klehs S, Schneider HE, Paul T (2017): Effects of Triple Cryoenergy Application on Lesion Formation and Coronary Arteries in the Developing Myocardium. Pediatr Cardiol $\underline{38}, 663-668$ 


\section{Inhaltsverzeichnis}

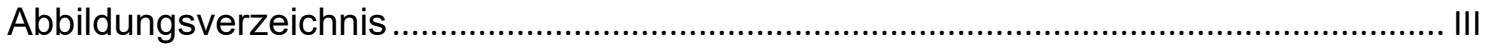

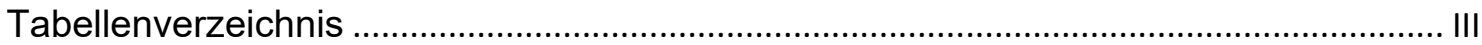

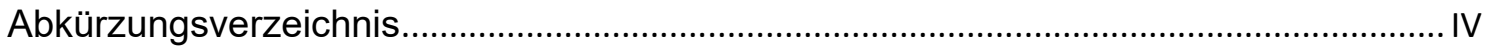

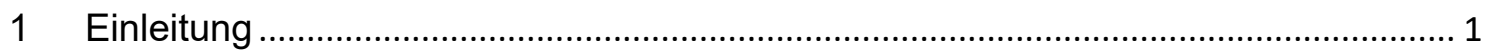

1.1 Das spezifische Erregungsbildungs- und Leitungssystem des Herzens............ 1

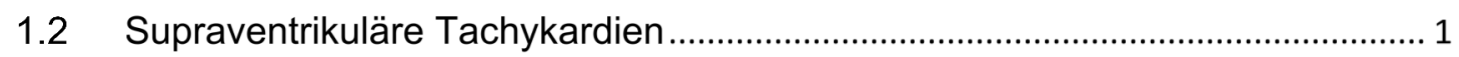

1.3 Pathomechanismen der supraventrikulären Tachykardie ................................... 3

1.4 Katheterablation von supraventrikulären Tachykardien.......................................... 5

1.5 Historischer Rückblick ......................................................................................... 10

1.6 Ablationsverfahren als Therapie von supraventrikulären Tachykardien .......... 11

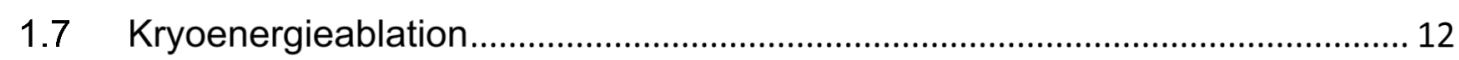

1.8 Komplikationen nach Katheterablation.............................................................. 14

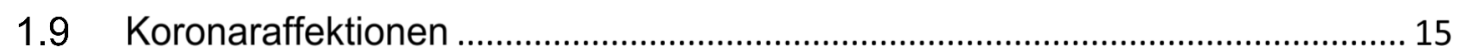

1.10 latrogen hervorgerufene Herzrhythmusstörungen............................................. 16

1.11 Fragestellung und Zielsetzung ....................................................................... 17

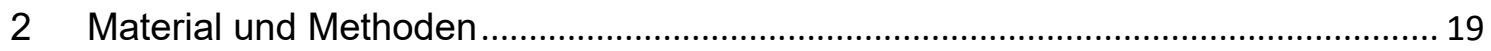

2.1 Vorbereitung, Voruntersuchungen und Anästhesie........................................... 19

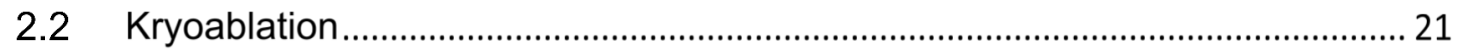

2.3 Nachuntersuchung $48 \mathrm{~h}$ nach dreifacher Kryoenergieapplikation..................... 22

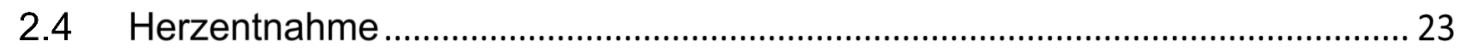

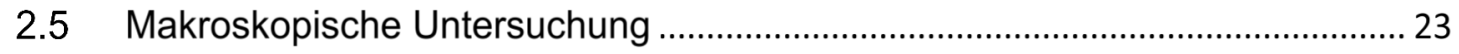

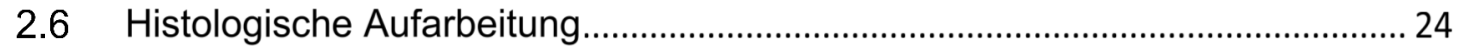

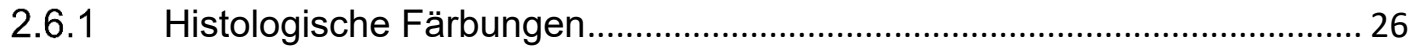

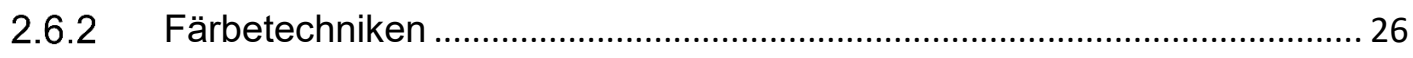

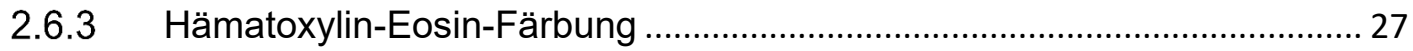

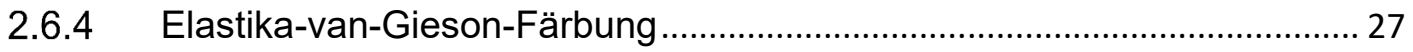

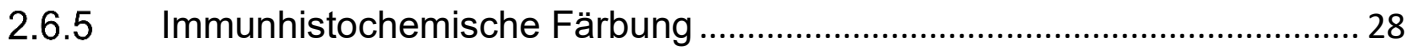

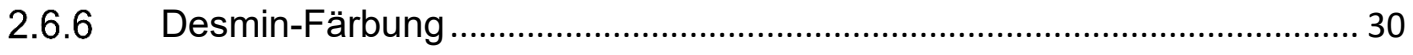

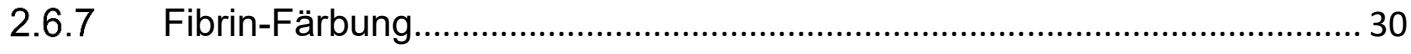

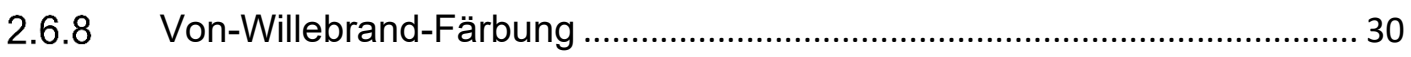

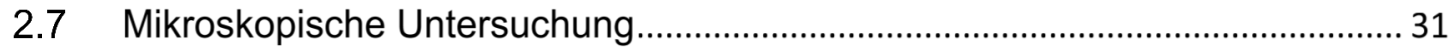

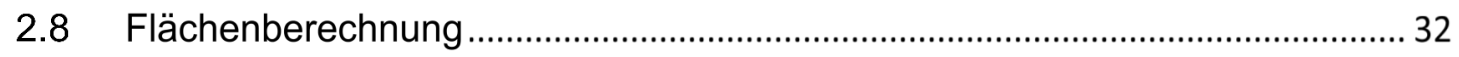

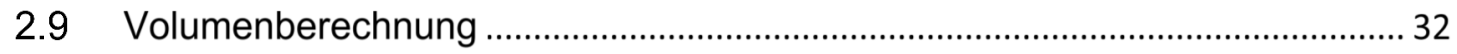

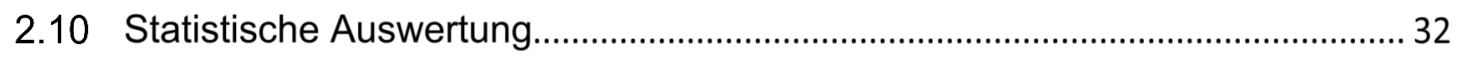

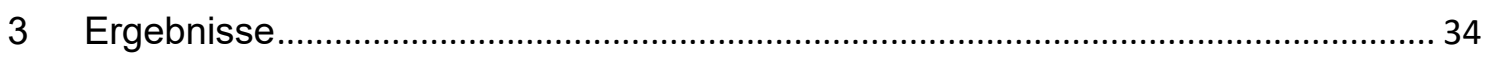




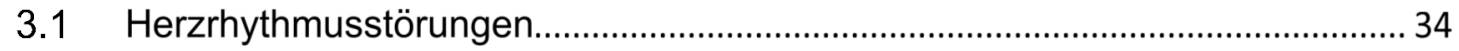

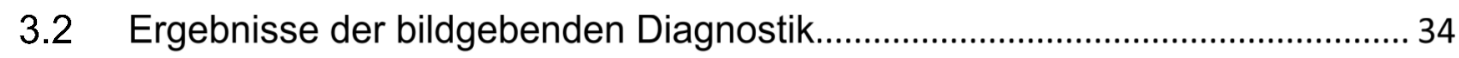

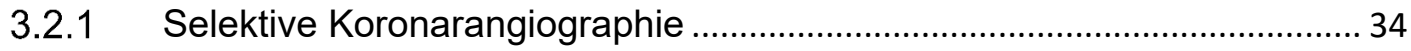

3.2.2 Intrakoronare Ultraschalluntersuchung (ICUS) ......................................... 35

3.3 Makroskopische Ansicht der Läsionen ................................................................... 36

3.4 Mikroskopische Ansicht der Läsionen .............................................................. 37

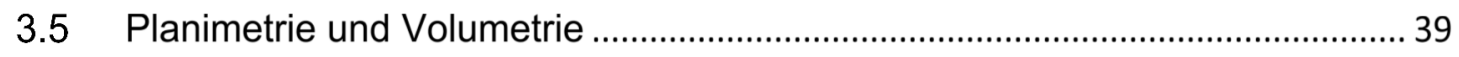

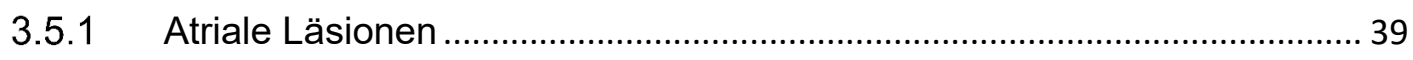

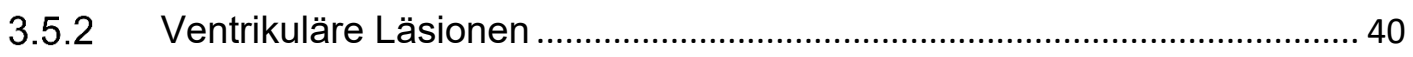

3.6 Vergleich von atrialen und ventrikulären Läsionsgrößen.................................... 41

3.7 Vergleich der Läsionsvolumina nach dreifacher Kryoenergieapplikation zu Läsionsvolumina nach doppelter Kryoablation................................................... 42

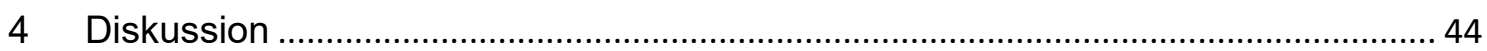

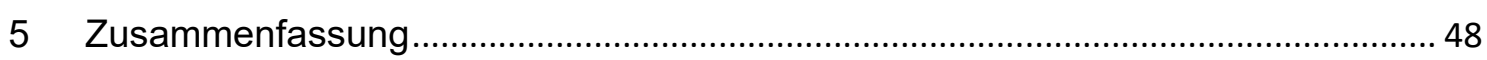

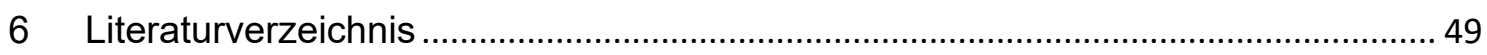

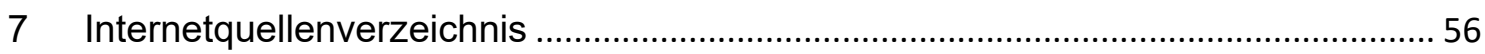




\section{Abbildungsverzeichnis}

Abbildung 1: Orthodrome und antidrome Reizweiterleitung ....................................... 4

Abbildung 2: WPW-Syndrom, Präexzitation im Sinusrhythmus .....................................4

Abbildung 3: Entstehung der AVNRT ...................................................................... 5

Abbildung 4: Schematische Darstellung Katheterablation und Gegenelektrode........... 12

Abbildung 5: Schematische Darstellung des Anfrierprozesses ................................... 13

Abbildung 6: Koronar-Stenose, nach Hochfrequenzstromapplikation .......................... 15

Abbildung 7: Ventrikuläre Tachykardie nach Ablation einer supraventrikulären

Tachykardie ................................................................................... 17



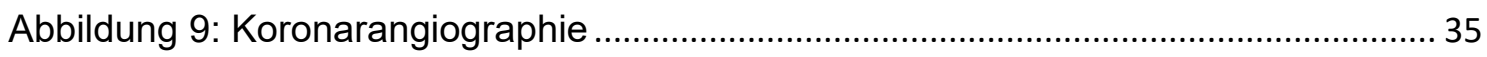

Abbildung 10: Intracoronare Ultraschalluntersuchung.................................................. 36

Abbildung 11: Makroskopische Ansicht einer Kryoläsion ........................................... 37

Abbildung 12: Transmurale Kryoläsion mikroskopisch (Atrium) .................................. 38

Abbildung 13: Transmurale Kryoläsion mikroskopisch (Ventrikel) ............................... 39

Abbildung 14: Box-Plot der Läsionsvolumina ............................................................ 42

\section{Tabellenverzeichnis}

Tabelle 1: Arbeitsschritte zur Fixierung mit aufsteigender Alkoholreihe ....................... 25

Tabelle 2: Arbeitsschritte zur Rehydrierung mit absteigender Alkoholreihe .................. 26

Tabelle 3: Arbeitsschritte zur Anfertigung der Hämatoxylin-Eosin-Färbung .................. 27

Tabelle 4: Arbeitsschritte zur Anfertigung der Elastika-van-Gieson-Färbung............... 27

Tabelle 5: Arbeitsschritte der Entplastung.............................................................. 28

Tabelle 6: Arbeitsschritte des allgemeinen immunhistochemischen Färbeprotokolls ... 29

Tabelle 7: Arbeitsschritte der Dehydrierung nach histologischer Färbung.................... 31

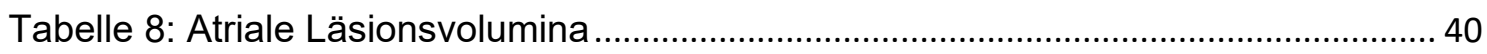

Tabelle 9: Ventrikuläre Läsionsvolumina .................................................................. 41

Tabelle 10: Vergleich der Läsionsvolumina von doppelter und dreifacher Kryoenergie43 


\section{Abkürzungsverzeichnis}

$\begin{array}{ll}\text { A. } & \text { Arteria } \\ \text { AK } & \text { Antikörper } \\ \text { AV } & \text { atrio-ventrikulär } \\ \text { AVNRT } & \text { atrio-ventrikuläre-nodale-Reentry-Tachykardie } \\ \text { AVRT } & \text { Atrio-ventrikuläre-Reentry-Tachykardie } \\ \text { DAB } & \text { Diaminobenzidin } \\ \text { EKG } & \text { Elektrokardiogramm } \\ \text { EPU } & \text { elektrophysiologische Untersuchung } \\ \text { F } & \text { French } \\ \text { H.E. } & \text { Hämatoxylin-Eosin } \\ \text { HF } & \text { Hochfrequenz } \\ \text { ICUS } & \text { intrakoronarer Ultraschall } \\ \text { IE } & \text { internationale Einheit } \\ \text { Kryo } & \text { Kryoenergie } \\ \text { LAO } & \text { linksanteriore schräge Projektion } \\ \text { LCA } & \text { linke Koronararterie } \\ \text { MK } & \text { Mitralklappe } \\ \text { RAO } & \text { rechtsanteriore schräge Projektion } \\ \text { RCA } & \text { rechte Koronararterie } \\ \text { RFA } & \text { Radiofrequenzstrom-Ablation } \\ \text { RIVA } & \text { Ramus interventrikularis anterior } \\ \text { SpO } 2 & \text { pulsoxymetrische Sauerstoffsättigung } \\ \text { SVT } & \text { supraventrikuläre Tachykardie } \\ \text { TK } & \text { Trikuspidalklappe } \\ \text { TTBS } & \text { Tris-Puffer-Lösung } \\ \text { V. } & \text { Vena } \\ \text { VT } & \text { ventrikuläre Tachykardie } \\ \text { WPW } & \text { Wolff-Parkinson-White } \\ & \end{array}$




\section{Einleitung}

\subsection{Das spezifische Erregungsbildungs- und Leitungssystem des Herzens}

Das Herz besitzt ein spezielles System zur Bildung und Leitung von elektrischen Impulsen, die von den Herzmuskelzellen in mechanische Arbeit umgesetzt werden und die Herzkontraktion bewirken.

Physiologischer Taktgeber des Herzens ist der Sinusknoten. Der Sinusknoten ist ein von dem umliegenden Gewebe anatomisch schwer abgrenzbares Konglomerat aus spezifischen Kardiomyozyten im Bereich der Einmündung der Vena cava superior im sogenannten Sulcus terminales cordis in den rechten Vorhof. Die spezifischen Kardiomyozyten besitzen die Eigenschaft, spontan überschwellig zu depolarisieren und so ein Aktionspotenzial zu erzeugen. Der Sinusknoten bildet in Ruhe etwa 60 bis 80 elektrische Impulse pro Minute. Diese elektrischen Impulse werden durch die Kardiomyozyten des Vorhofmyokards zum Atrioventrikularknoten (AV-Knoten) fortgeleitet. Der AV-Knoten liegt im Koch-Dreieck im inferioren rechten Vorhof, welches durch das Ostium des Coronarvenensinus, das septale Segel der Trikuspidalklappe und die Todarosehne (eine Verlängerung der Valvula venae cavae inferioris im Bereich der Einmündung der unteren Hohlvene in den rechten Vorhof) eingegrenzt wird. Durch den AV-Knoten und das sich anschließende His-Bündel wird das elektrisch nichtleitende, aus Bindegewebe bestehende Herzskelett überbrückt und die elektrischen Impulse können an das Ventrikelmyokard weitergeleitet werden. Charakteristisch ist die Leitungsverzögerung im Bereich des AV-Knotens. Diese Eigenschaft des spezifischen Reizleitungssystems wird als "dekrementale Leitung" bezeichnet. Die weitere Reizleitung erfolgt über die Tawara-Schenkel und Purkinje-Fasern in das Arbeitsmyokard des rechten und linken Ventrikels.

\subsection{Supraventrikuläre Tachykardien}

Supraventrikuläre Tachykardien (SVT) gehören zu den häufigsten symptomatischen Tachyarrhythmien im Kindes- und Jugendalter (Kriebel et al. 2007a, Saul et al. 2016). Von einer SVT spricht man, wenn der Ursprung der Tachykardie bzw. der kritische Bestandteil des Wiedereintrittsmechanismus der Tachykardie vorhofseitig der Aufzweigung des His-Bündels in die Tawara-Schenkel lokalisiert 
ist. Abhängig vom Entstehungsmechanismus können grundsätzlich zwei Arten von SVT unterschieden werden.

1. Wiedereintritts- (Reentry-) Mechanismus: hier liegen mindestens zwei elektrisch leitende Zonen mit unterschiedlichen Leitungsgeschwindigkeiten und Refraktärzeiten vor, über welche dann eine Reentry-Tachykardie entstehen kann. Beispiel hierfür sind akzessorische atrioventrikuläre Leitungsbahnen (s.u.).

2. Erhöhte Automatie: hier finden sich neben dem Sinusknoten ein oder mehrere weitere pathologische Schrittmacherzentren im Bereich des Vorhofmyokards, welche kontinuierlich oder diskontinuierlich elektrische Impulse mit einer höheren Frequenz als die des Sinusknotens generieren und so zur Entstehung fokaler atrialer Tachykardien führen.

Die Prävalenz der SVT wird nach aktueller Studienlage mit 2,25/1000 Personen angegeben, wobei für Kinder unter 19 Jahren von einer Inzidenz von 13/100.000 ausgegangen wird. Die Dunkelziffer dürfte jedoch aufgrund der sporadisch auftretenden Symptomatik, einem häufigen spontanen Sistieren der Tachykardie im Säuglings- und Kleinkindalter und einem dadurch bedingten Ausbleiben der Diagnosestellung der SVT noch höher ausfallen (Brugada et al. 2013).

Die klinische Symptomatik ist abhängig vom Alter der Patienten, der Herzfrequenz während der SVT und der kardialen Anatomie. Bei ansonsten herzgesunden Säuglingen kommt es aufgrund der extrem hohen Kammerfrequenzen während der Tachykardie von bis zu 300 Schlägen pro Minute und einer dadurch bedingten verkürzten diastolischen Füllung der Ventrikel rasch zur Entwicklung einer Herzinsuffizienz mit vermehrtem Schwitzen, Nahrungsverweigerung, Erbrechen, sowie pulmonaler und intestinaler Stauung. Häufig werden diese Kinder mit dem Verdacht auf eine Pneumonie in die Klinik eingewiesen. Bei größeren Kindern und Jugendlichen ändert sich die Symptomatik dahingehend, dass eher Palpationen, Schwindel, Unwohlsein und allgemeine körperliche Erschöpfung im Vordergrund stehen. Synkopen sind hingegen selten. Die chronisch-permanenten Formen supraventrikulärer Tachykardien gehen aufgrund der nicht allzu stark erhöhten Herzfrequenzen seltener mit akuten Symptomen einher. Allerdings kommt es häufig, wie schon oben beschrieben, durch die permanent gesteigerte 
Herzfrequenz zu einer Tachykardie-induzierten Kardiomyopathie (Paul et al. 2018).

\subsection{Pathomechanismen der supraventrikulären Tachykardie}

An dieser Stelle soll nur auf die beiden häufigsten Formen der SVT bei Kindern und Jugendlichen eingegangen werden. Die häufigste Form der SVT im Kindesalter stellt die atrioventrikuläre Reentry-Tachykardie (AVRT) dar. Hierbei kommt es, bedingt durch das Vorhandensein einer akzessorischen atrioventrikulären Leitungsbahn (s. Abbildung 1), zu einer kreisenden Erregung zwischen Atrium und Ventrikel. Die Richtung der Reizübertragung zwischen Atrium und Ventrikel kann orthodrom oder antidrom erfolgen. Bezugspunkt für die Nomenklatur ist jeweils die Richtung der Leitung über das spezifische Reizleitungssystem des Herzens (AV-Knoten und His-Bündel). Bei der orthodromen Form kommt es zu einer antegraden Überleitung zwischen Atrium und Ventrikel über den AV-Knoten und einer anschließenden retrograden Leitung zurück in den Vorhof über die akzessorische Leitungsbahn. Die Kammerkomplexe im EKG sind in Tachykardie schmal. Bei der antidromen Form hingegen kommt es zunächst zu einer antegraden Erregungsüberleitung vom Vorhof in die Kammer über die akzessorische Leitungsbahn und anschließend zu einer retrograden Rückleitung aus der Kammer in den Vorhof über den AV-Knoten. Bei der antidromen Form der SVT sind die Kammerkomplexe breit. Von einem Wolff-Parkinson-White-Syndrom (WPWSyndrom) wird gesprochen, wenn bei Patienten mit SVT aufgrund einer akzessorischen atrioventrikulären Leitungsbahn im Sinusrhythmus eine antegrade (d. h. vom Atrium auf den Ventrikel) Erregungsleitung über die akzessorische Bahn vorliegt und zu einem Präexzitationsmuster im Oberflächen-EKG führt (s. Abbildung 2). 


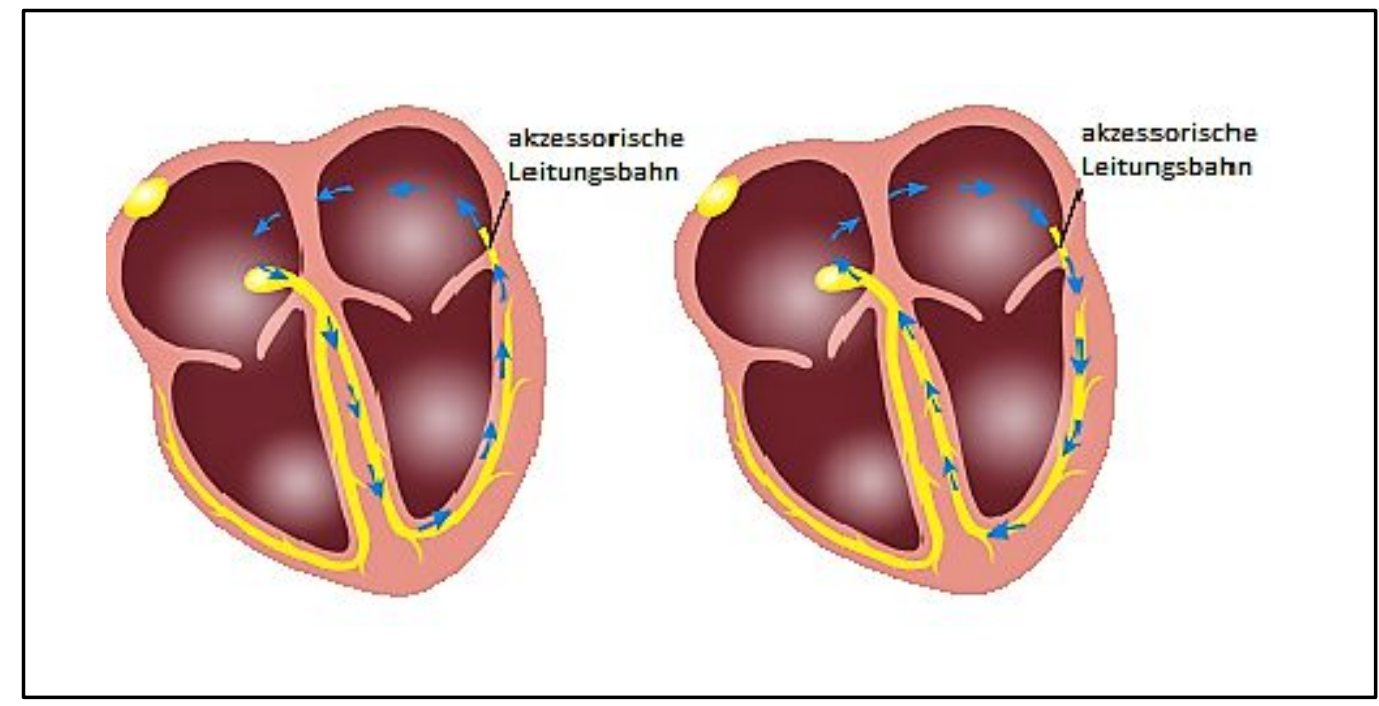

Abbildung1: Orthodrome (links) und antidrome (rechts) SVT über eine akzessorische Leitungsbahn (www.ecgpedia.org, OCT und ACT, CC BY-NC-SA 3.01)

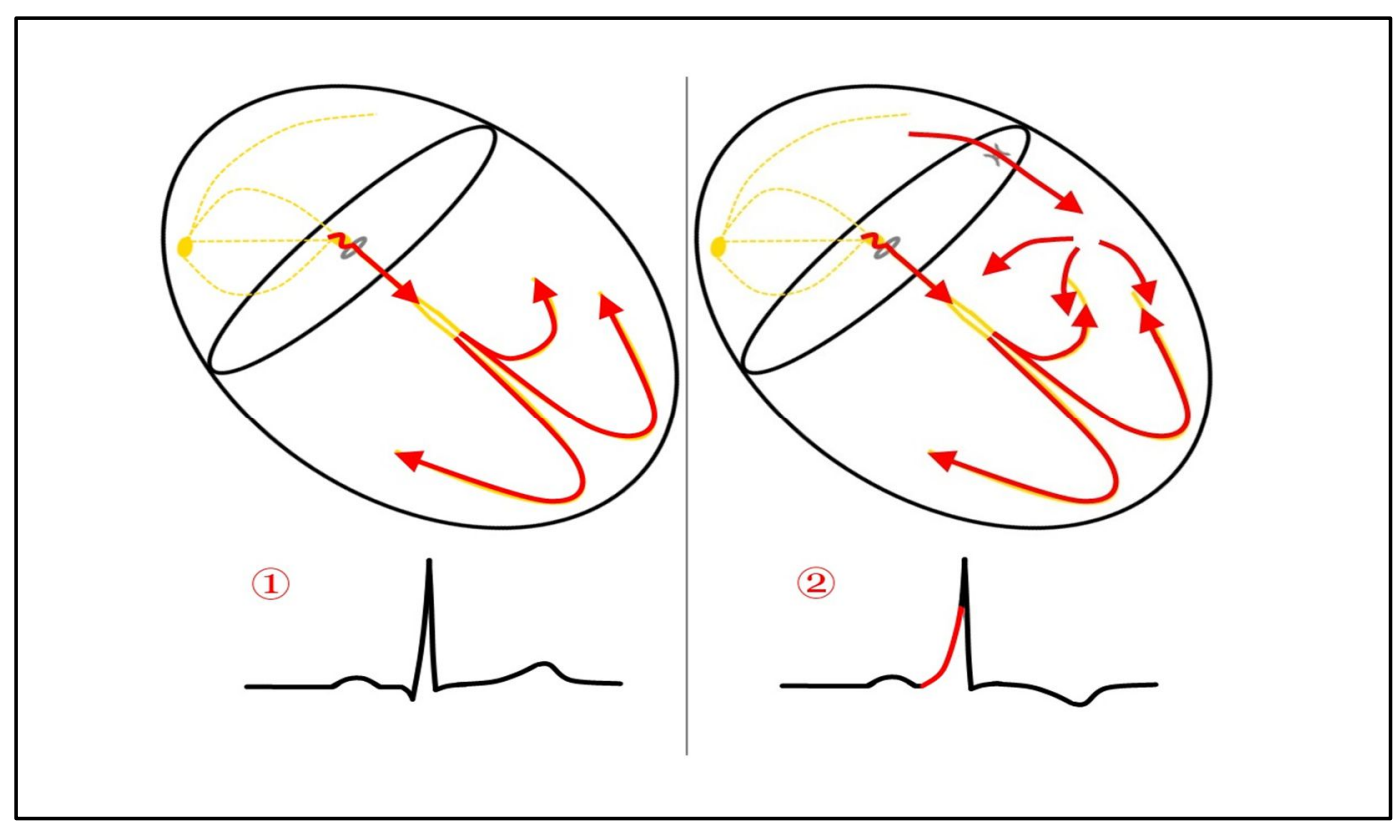

Abbildung 2: WPW-Syndrom, Präexzitation im Sinusrhythmus (www.ecgguru.com, Christopher Watford, CC BY-NC-SA 3.0 )

Bei der AV-Knoten-Reentry-Tachykardie (AVNRT) liegen unterschiedliche elektrophysiologische Eigenschaften im AV-Knoten selber vor, welche zu einer

\footnotetext{
${ }^{1}$ https://creativecommons.org/licenses/by-nc-sa/3.0/

${ }^{2}$ https://creativecommons.org/licenses/by-nc-sa/3.0/
} 
funktionellen Spaltung und Unterschieden in der Leitungsgeschwindigkeit und Refraktärzeit der beiden funktionellen AV-Knotenschenkel führen. Es lassen sich elektrisch/funktionell ein langsam leitender und ein schnell leitender Schenkel der dualen AV-Knotenleitungsbahnen unterscheiden. Im Gegensatz zu einer akzessorischen Leitungsbahn liegt der funktionellen Spaltung des AV-Knotens aber kein eindeutig anatomisch abgrenzbares Korrelat zugrunde. Während der schnell leitende Schenkel eine lange Refraktärzeit aufweist, ist die Refraktärzeit des langsam leitenden Schenkels kurz. So kann, z. B. durch ventrikuläre oder supraventrikuläre Extrasystolen induziert, eine AV-Knoten-Reentry-Tachykardie entstehen (s. Abbildung 3). Bei Adoleszenten ist die AV-Knoten-Reentry-Tachykardie die häufigste Ursache paroxysmaler supraventrikulärer Tachykardien.

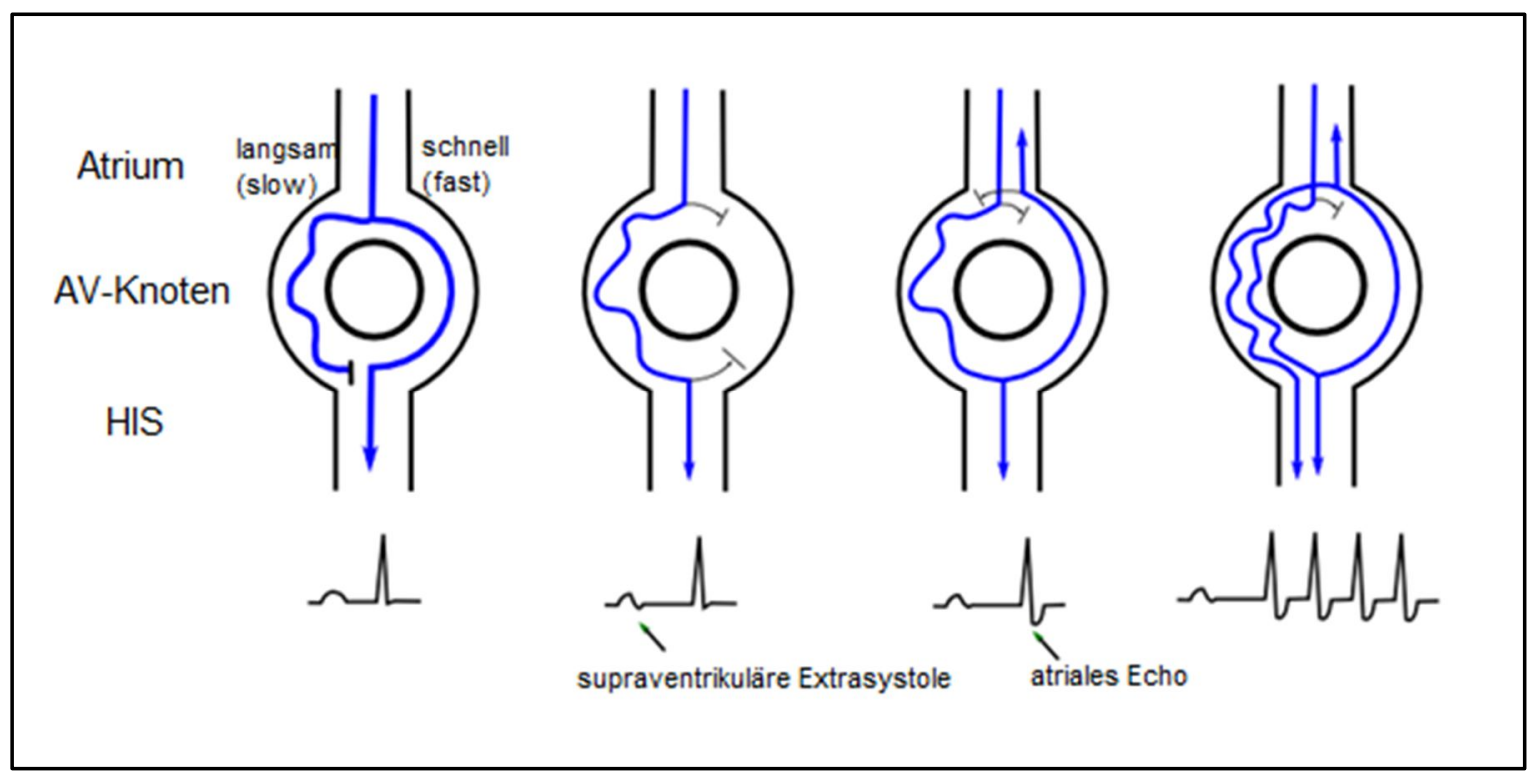

Abbildung 3: Entstehung der AVNRT (www.ecgpedia.org, Re-entry, CC BY-NC-SA 3.0 ${ }^{3}$ )

\subsection{Katheterablation von supraventrikulären Tachykardien}

Supraventrikuläre Tachykardien aufgrund akzessorischer atrioventrikulärer Leitungsbahnen oder aufgrund dualer Leitungseigenschaften des AV-Knotens sind die häufigsten Ursachen für paroxysmales Herzrasen im Kindes- und Jugendalter. Auch wenn es sich in der Regel nicht um unmittelbar lebensbedrohliche Herzrhythmusstörungen handelt, besteht dennoch die Indikation zur Therapie, zum Beispiel bei symptomatischen oder potentiell lebensbedrohlichen tachykarden

\footnotetext{
${ }^{3}$ https://creativecommons.org/licenses/by-nc-sa/3.0/
} 
Herzrhythmusstörungen. Grundsätzlich bestehen zwei Möglichkeiten der Therapie der SVT bzw. des Substrats: 1. Symptomatische medikamentöse Therapie und 2. kausale Therapie durch Katheterablation des Tachykardiesubstrates. Grundsätzlich wird bei Kindern mit einem Körpergewicht von > 15 kg die Katheterablation empfohlen (Paul et al. 2018).

Die Radiofrequenzstrom-Ablation (RFA) des anatomischen Substrates von supraventrikulären Tachykardien ist bei Kindern aufgrund des niedrigen Risikos und der hohen Effektivität ein etabliertes Behandlungsverfahren. Mittels in die Herzhöhlen eingebrachter Elektrodenkatheter werden pathologische, elektrisch leitende Strukturen identifiziert und durch die Applikation von Radiofrequenzstrom verödet. Den Patienten kann durch dieses kurative Behandlungsverfahren die lebenslange Einnahme von antiarrhythmischen Medikamenten erspart werden. Allerdings zeigten mehrere Studien, dass ein Körpergewicht von $\leq 15 \mathrm{~kg}$ mit einer höheren Komplikationsrate nach Radiofrequenzstrom-Ablation assoziiert ist (Kugler et al. 1997, Kugler et al. 1994, Schaffer et al. 2000). Zusätzlich belegten experimentelle und klinische Untersuchungen, dass die Radiofrequenzstrom-Applikation am unreifen Myokard mit signifikanten späten Gewebeeffekten assoziiert sein kann. Bei neugeborenen Lämmern wurde zudem eine Zunahme der Läsionsgröße nach Radiofrequenzstrom-Applikation im Laufe des weiteren Wachstums beobachtet (Saul et al. 1994). Auch eine Affektion der Koronararterien im Sinne von Stenosen konnte sowohl im kurzfristigen als auch im mittelfristigen Verlauf nach Radiofrequenzstrom-Applikation im Tiermodell nachgewiesen werden (Paul et al. 1997, Bökenkamp et al. 2000). Aber nicht nur im Tiermodell sondern auch bei Kindern und Erwachsenen wurden verschiedene Fallberichte von Koronararterienverengungen und sogar Koronararterienverschlüssen nach Radiofrequenzstrom-Applikation publiziert (Bertram et al. 2001, Strobel et al. 2001, Khanal et al. 1999, Chatelain et al. 1995, Pons et al. 1997, de Paola et al. 2003). Somit konnten die tierexperimentell gewonnenen Daten auch klinisch bestätigt werden. Kugler et al. berichteten von einem Todesfall eines 13-jährigen Patienten durch den Verschluss der linken Koronararterie drei Stunden nach Radiofrequenzstrom-Applikation (Kugler et al. 2002).

Mehrere klinische Studien zeigten, dass die Kryoenergie eine sichere und effektive alternative Methode zur Ablation von arrhythmogenen Substraten bei Kindern, Jugendlichen und Erwachsenen ist (Lowe et al. 2003, Rodriguez et al. 
2002, Riccardi et al. 2003, Lanzotti et al. 2002, Kirsh et al. 2005, LaPage et al. 2011, Kimman et al. 2004). Vorteile der Kryoenergie sind eine stabilere Katheterposition durch das Anfrieren der Katheterspitze am Myokard (Kryoadhäsion) und die Möglichkeit einer Überprüfung des Ablationserfolges vor Induktion einer permanenten Läsion durch das sog. Kryomapping. In Bereichen mit dem erhöhten Risiko eines AV-Blockes ist vor Induktion einer permanenten Läsion auch die Überprüfung der Leitungseigenschaften des AV-Knotens während des Kryomappings möglich (Skanes et al. 2000). Ein permanenter AV-Block nach Ablation eines Tachykardiesubstrates mittels Kryoenergie ist bislang nicht publiziert. Zusätzlich zu diesen klinischen Daten konnte in einer experimentellen Studie an erwachsenen Hunden demonstriert werden, dass die Applikation von Kryoenergie im Vergleich zur Radiofrequenzstrom-Applikation eine höhere Sicherheit bezüglich der Induktion von Koronararterienstenosen besitzt (Aoyama et al. 2005). Die Größe der durch Kryoablation induzierten Läsionen ist dabei multifaktoriell bedingt. Die Größe der gewählten Katheterspitze, die Zieltemperatur, mit der abladiert wird, und die Dauer der Ablation zählen zu den Faktoren die die Läsionsgröße beeinflussen (Markovitz et al. 1988).

In tierexperimentellen Untersuchungen zur Kryoablation wurden kleinere und schärfer demarkierte Läsionen nach einmaliger Kryoablation am unreifen Myokard von Schweinen im Vergleich zur Radiofrequenzstrom-Ablation dokumentiert. Zudem wurden bislang keine signifikanten Koronararterienaffektionen nach Kryoenergie-Applikation beschrieben (Kriebel et al. 2009, Schneider et al. 2009). In der klinischen Praxis sind jedoch eine geringere akute Erfolgsrate (70-80 \%) und eine höhere Rezidivrate (bis zu 40 \%) nach Kryoenergie-Ablation im Vergleich zur Radiofrequenzstrom-Applikation (95-98 \%) sowohl für AV-Knoten-Reentry-Tachykardien (AVNRT) als auch für akzessorische Leitungsbahnen zu beobachten (Kriebel et al. 2005, Kirsh et al. 2005, Bar-Cohen et al. 2006, Drago et al. 2005, Miyazaki et al. 2005a, Collins et al. 2007). Daher wird die Kryoenergie überwiegend zur Ablation von Substraten in Regionen eingesetzt, die mit einem erhöhten Risiko einer Affektion der Herzkranzgefäße oder eines AV-Blocks behaftet sind.

Um die Erfolgsrate der Ablation durch Kryoenergie-Applikation zu erhöhen, haben einige Arbeitsgruppen ein alternatives Konzept in Form von, Sicherheitsläsionen' klinisch angewandt. Hierbei wird eine zusätzliche Läsion am selben Ort 
durch einen Zyklus von Anfrieren-Auftauen-Anfrieren mit der Erwartung eingesetzt, dass durch größere Läsionen eine Steigerung der akuten Effektivität und eine Reduktion der Rezidivrate erzielt werden kann. Durch dieses Konzept ließ sich die Erfolgsrate bis auf 97,4 \% steigern und die Rezidivrate auf 6,8 \% senken (Rodriguez et al. 2002, Skanes et al. 2000, Collins et al. 2007, Drago et al. 2010). In einer tierexperimentellen Studie konnte gezeigt werden, dass diese modifizierte Form der Kryoenergie-Applikation nicht zu akuten Verengungen der Koronararterien führt. Die feingewebliche Untersuchung der behandelten Herzen zeigte zwar geringe Veränderungen der Tunica adventitia und media der Koronargefäßwand, das sensible Gefäßendothel war aber bei keinem der untersuchten Tiere beschädigt. Im Vergleich zu den durch Radiofrequenzstrom induzierten Gefäßveränderungen ließ sich somit auch mit der modifizierten Form der Kryoenergie-Applikation (Anfrier-Auftau-Anfrier-Zyklus) ein günstigeres Ergebnis bezüglich der Veränderungen der Koronararterien dokumentieren. Hinsichtlich der morphometrisch bestimmten Läsionsgröße ergaben sich unterschiedliche Ergebnisse. Verglichen mit der einmaligen Applikation von Kryoenergie konnte nach zweimaliger Kryoenergie-Applikation eine Zunahme der Läsionsgröße (Volumen) im Bereich der Vorhöfe dokumentiert werden, während sich die Läsionsvolumina im Bereich der Ventrikel nicht unterschieden. Auch die Tiefe der durch zweimalige Kryoenergie-Applikation erzielten Läsionen unterschied sich nicht signifikant im Vergleich mit Läsionen durch die einmalige Applikation von Kryoenergie. Diese Daten beziehen sich auf kurzfristige Veränderungen nach KryoenergieApplikation (Schneider et al. 2012). Auch die späte Untersuchung der myokardialen Läsionen sowie der Koronararterien sechs Monate nach zweimaliger Kryoenergie-Applikation zeigte keine signifikanten Stenosen der Koronararterien oder eine Zunahme der Läsionsgröße mit dem Wachstum der Tiere (Schneider et al. 2013).

Aufgrund dieser Daten lässt sich schlussfolgern, dass die zweimalige Kryoenergie-Applikation eine sichere und effektive Methode zur Ablation von Substraten supraventrikulärer Tachykardien darstellt. Die aus anderen Veröffentlichungen bislang erzielten Schlussfolgerungen einer Größen- bzw. Tiefenzunahme, und daraus abgeleitete Angaben zur Erfolgsrate, aus Ablationserfolgen nach Anwendung des Anfrier-Auftau-Anfrier-Zyklus der Kryoenergie aus klinischen und tierexperimentellen Beobachtungen waren aber widersprüchlich (Dubuc et al. 1999, 
Avitall et al. 2004, Manusama et al. 2004). Auch entsprachen die z. T. verwendeten Kryoablationskatheter und -temperaturen nicht den aktuell klinisch eingesetzten Kathetertechniken.

Unter der Annahme, dass eine weitere Erhöhung der Anzahl der Anfrier-AuftauZyklen den akuten und langfristigen Erfolg der Ablation eines Tachykardiesubstrates mittels Kryoenergie verbessert, haben Gist et al. ein modifiziertes Kryoenergie-Protokoll mit drei konsekutiven Anfrier-Auftau-Zyklen bei der Ablationstherapie linksseitig lokalisierter akzessorischer Leitungsbahnen klinisch erfolgreich angewandt (Gist et al. 2009). Die Arbeitsgruppe konnte zeigen, dass durch die modifizierte Form der Kryoenergie-Applikation mit drei aufeinanderfolgenden Zyklen der akute und mittelfristige Ablationserfolg ähnlich hoch wie bei der Verwendung von Radiofrequenzstrom war. Während der Nachbeobachtung der Patienten lag die Rezidivrate nach zwölf Monaten bei $<5 \%$ und somit deutlich unter den bisher publizierten Daten (Kriebel et al. 2005, Gaita et al. 2003). Die Herzkranzgefäße wurden bei den Patienten nicht untersucht, somit ist die Entwicklung subklinischer Koronarveränderungen nicht auszuschließen. Es ist nicht bekannt, wie sich die weitere Erhöhung der Anzahl der Anfrier-Zyklen auf das Myokard und die Herzkranzgefäße auswirkt. Da von den Autoren neben einer Erhöhung der Zahl der Anfrierzyklen auch zusätzliche Läsionen neben das identifizierte Tachykardiesubstrat platziert wurden, stellt sich die Frage, ob die hohe Effektivität des verwendeten Ablationsprotokolls durch eine Optimierung der punktuellen Läsion oder einen Summationseffekt mehrerer nebeneinander platzierter Läsionen zustande kam (Gist et al. 2009).

Ziel der vorliegenden Studie war es daher, durch exakte Volumetrie herauszufinden, ob tatsächlich mittels Kryoenergie-Applikation eine Größenzunahme der Läsionen durch einen dreifachen Anfrier-Auftau-Zyklus im Myokard erzielt werden kann (Paul et al. 1997, Bokenkamp et al. 2000, Kriebel et al. 2009, Schneider et al. 2012). Die im Rahmen der vorliegenden Studie gemessenen Läsionsgrößen sollten mit den Werten aus vorausgegangenen Studien zur einfachen und doppelten Kryoenergie-Applikation verglichen werden. Da in den vorausgegangenen Studien das gleiche Tiermodell und der gleiche Kryoenergie-Ablationskatheter verwendet worden waren, war das Heranziehen dieser „historischen“ Vergleichskohorte möglich. Da größere Läsionen auch ein potentiell höheres Risiko für unerwünschte Komplikationen, insbesondere eines AV-Blockes und einer Affektion 
von Herzkrangefäßen, mit sich bringen können, galt es, diese Faktoren im Tiermodell systematisch zu analysieren. Bislang galt die Kryoenergie hinsichtlich solcher Komplikationen gegenüber der Radiofrequenzstrom-Applikation als überlegen.

\subsection{Historischer Rückblick}

Die ersten Ansätze zur Therapie von tachykarden Herzrhythmusstörungen bestand in der Anwendung von vagalen Manövern und der Gabe von Digitalispräparaten. Aus den 70er Jahren des 20. Jahrhunderts datiert der erste kurative Therapieansatz, der aus einem offenen chirurgischen Eingriff am Herzen und der Entfernung des anatomischen Substrats, einer akzessorischen atrioventrikulären Leitungsbahn, bestand (Sealy et al. 1976). Diese Form der chirurgischen Therapie und der Unterbrechung der akzessorischen Leitungsbahnen mit dem Skalpell wurde im Laufe der Jahre weiterentwickelt. So war es bald möglich, die akzessorischen Bahnen mittels epicardialem Mapping (Sealy et al. 1981) genau zu lokalisieren, und diese durch das Resezieren von Gewebebrücken (d.h. der akzessorischen Leitungsbahn) im Bereich des Anulus fibrosus zu entfernen. Obwohl dieser Ansatz sehr effektiv war, konnte er sich langfristig nie als Therapie der Wahl durchsetzen. Ursächlich dafür war die hohe Invasivität des Verfahrens: die Notwendigkeit der Sternotomie und der Anwendung der Herz-Lungen-Maschine und die damit assoziierte Sterblichkeit mit Mortalitätsraten von bis zu 5 \% (Cox et al. 1985, Ruskin 1991).

Der Beginn der Katheterablationstherapie datiert auf das Jahr 1979, als bei einer elektrophysiologischen Untersuchung versehentlich Gleichstrom im Bereich des His-Bündels appliziert wurde und ein persistierender kompletter AV-Block resultierte (Vedel et al. 1979).

Die erste Katheterablation im Kindesalter erfolgte im Jahr 1983. Bei zwei Kindern mit einer junktionalen ektopen Tachykardie wurde eine His-Bündelablation mit Gleichstrom durchgeführt (Gillette et al. 1983). Die erste Katheterablation einer akzessorischen Leitungsbahn erfolgte an der Universitätsklinik Göttingen ebenfalls im Jahr 1983 durch Helmut Weber und Lothar Schmitz (Weber und Schmitz 1983). Die erste Radiofrequenzstrom-Ablation bei einem Kind erfolgte 1990 durch George van Hare in San Francisco (van Hare et al. 1990). 
1986 beobachtete die Arbeitsgruppe um Lavergne, dass es bei der Kardioversion am Tiermodell durch die Stromapplikation zu Myokardläsionen kam, die Auswirkungen auf die Erregungsausbreitung hatten (Lavergne et al. 1986). Borggrefe griff diese Beobachtung auf und entwickelte eine Technik, mit der es inm 1987 erstmals gelang, eine akzessorische atrio-ventrikuläre Leitungsbahn mittels Radiofrequenzenergie zu unterbrechen (Borggrefe et al. 1987).

Seit 2003 wird die Kryoenergieablation als Therapiealternative zur RFA bei verschiedenen arrhythmogenen Substraten verwendet (Collins et al. 2011). Die ersten Veröffentlichungen zum Einsatz der Kryoablation waren vielversprechend, jedoch zeichnete sich schnell eine wesentlich höhere Rezidivrate ab, als bei der RFA (Kriebel et al. 2005, Miyazaki et al. 2005a, BarCohen et al. 2006, Collins et al. 2007, Drago et al. 2010). Allerdings zeigte sich im Laufe der Jahre, dass die Rezidivrate umgekehrt proportional zur Erfahrung des anwendenden Arztes im Umgang mit der neuen Technologie war. Neuere Veröffentlichungen zeigen eine Abnahme der Rezidivrate bei zunehmender Erfahrung und Sicherheit des Anwenders im Umgang mit dem Verfahren (LaPage et al. 2010, Silver et al. 2010, Czosek et al. 2010, Collins et al. 2011). Nun da die Kryoenergieabaltion schon über 15 Jahre angewandt wird, sind die Erfahrungen der Handhabung, des Nutzen und die Kenntnisse der Limitationen ausreichend, um es als sichere und effektive Alternative zur RFA anwenden zu können (Kriebel et al. 2005, 2007b, Miyazaki et al. 2005 a, LaPage et al. 2010, Collins et al. 2011). Allerdings gilt es, durch Studien, wie die Vorliegende, diese Ablationstechnik zu optimieren, um einen für den Patienten maximalen Nutzen aus der Kryoenergieablation zu ziehen.

\subsection{Ablationsverfahren als Therapie von supraventrikulären Tachykardien}

Goldstandard zur Ablation von Substraten supraventrikulärer Tachykardien ist die Anwendung von Radiofrequenzstromenergie (RF-Energie). RF-Energie wird hierfür mit hoher Sicherheit und Effektivität eingesetzt (Brugada et al. 2013, Morady 2004)

Über einen Katheter wird Hochfrequenzstrom im Radiofrequenzbereich von 300$500 \mathrm{kHz}$ an das umliegende Myokard abgegeben und erzeugt durch Wärmeentwicklung eine Koagulationsnekrose. Der Radiofrequenzstrom fließt hierbei durch 
den Körper des Patienten zu einer neutralen Gegenelektrode, die beispielweise am Rücken des Patienten angebracht wird (s. Abbildung 4). Die Koagulationsnekrose führt zum Untergang des Tachykardiesubstrates. An der Stelle des Gewebeuntergangs bildet sich eine Narbe aus elektrisch inertem Bindegewebe.

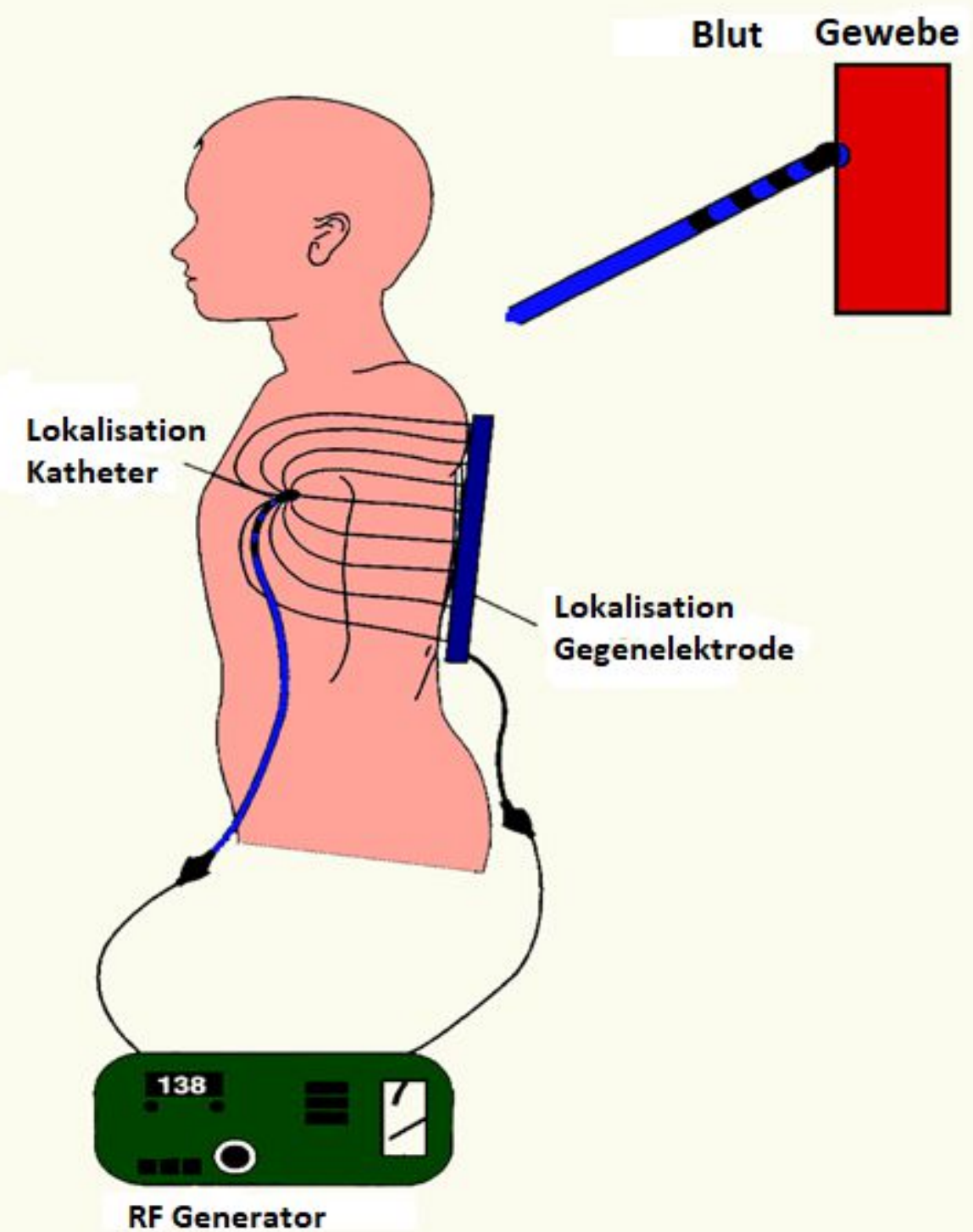

Abbildung 4: Schematische Darstellung Radiofrequenz-Katheterablation und Gegenelektrode (Mit freundlicher Genehmigung durch Herrn Prof. Dr. med. T. Paul)

\subsection{Kryoenergieablation}

Im Gegensatz zur RF-Energie wird bei der Anwendung von Kryoenergie die Ablationskatheterspitze durch das Einleiten von flüssigem Stickstoff auf eine Temperatur von $-70{ }^{\circ} \mathrm{C}$ bis $-80^{\circ} \mathrm{C}$ abgekühlt (s. Abbildung 5). Die bei ausreichend langer Kryoenergieapplikation entstehende Nekrose führt dann zur irreversiblen Schädigung des Tachykardiesubstrates. Ein wesentlicher Vorteil der Kryoenergie 
ist, dass durch die Kälteapplikation bereits vor Entstehung einer irreversiblen Läsion der Effekt der Kryoenergie auf das Myokard und auch das spezifische Reizleitungssystem erkennbar ist. So kann die Kryoenergieapplikation bei einer Beeinträchtigung des spezifischen Reizleitungssystem unterbrochen werden, bevor eine irreversible Schädigung entsteht.

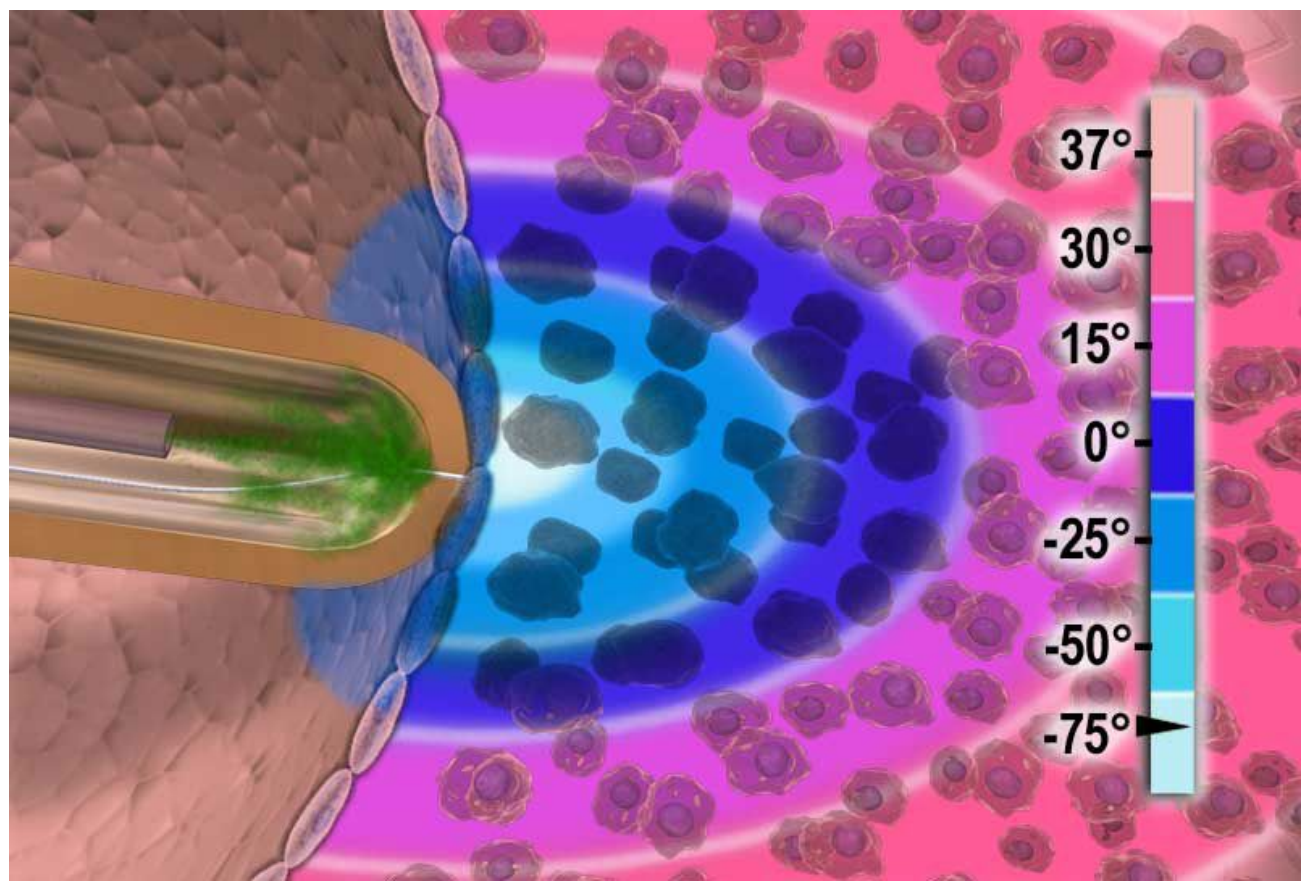

Abbildung 5: Schematische Darstellung des Anfrierprozesses bei der Kryoenergieablation. An der Katheterspitze kommt es zur sog. „Eisballformation“. (www. wikipedia.org, CC BY-SA 3.04)

Das Myokard reagiert auf die Läsionsinduktion durch Kryoenergie und das anschließende Auftauen mit Hämorrhagien, Inflammationsreaktionen und fibrösem Umbau (Holman et al. 1983, Lustgarten et al. 1999, Mazur 1970). Die detaillierten histopathologischen Auswirkungen und zellulären Reparationsmechanismen nach der Kryoenergieablation im sich entwickelnden Myokard sind noch nicht ausreichend bekannt und abschließend untersucht. In vorangegangenen tierexperimentellen Untersuchungen stellten sich die Läsionen nach Kryoenergie sowohl makroskopisch als auch mikroskopisch als glatt berandet dar, die sich deutlich von gesundem Myokardgewebe unterschieden (Kriebel et al. 2009, Schneider et al. 2013).

Die Heilungsprozesse nach Kryoenergieapplikation laufen schneller ab als nach Radiofrequenzstromapplikation. Dazu beschrieben Aoyama et al. einen Versuch

\footnotetext{
${ }^{4}$ https://creativecommons.org/licenses/by-sa/3.0/legalcode
} 
an 29 Hundeherzen, welche sowohl einer Radiofrequenzstromapplikation als auch einer Kryoenergieapplikation unterzogen wurden. Es zeigte sich in allen Präparaten der histopathologische Nachweis von transmuralen Nekrosen im Bereich der Koronarsinus (Aoyama et al. 2005). Allerdings konnte in den Läsionen eine Woche nach Kryoenergieapplikation, anders als nach Radiofrequenzstromapplikation, histologisch die Infiltration durch Fibroblasten nachgewiesen werden. Diese Beobachtung lässt auf einen schnelleren Heilungsvorgang nach Kryoenergieapplikation schließen. Selbe Beobachtung wurde ebenfalls in einer Arbeit von Skanes et al. beschrieben, welche in Kryoenergieläsionen ein homogeneres und intakteres Muskelgewebe beschrieb, ohne den für durch RFA induzierten typischen großflächigen nekrotischen Zelluntergang (Skanes et al. 2004). Diese Befunde trugen zum einen zur Erklärung der niedrigeren Komplikationsrate, zum anderen aber auch zur Erklärung der höheren Rezidivrate nach Kryoenergieablation bei.

\subsection{Komplikationen nach Katheterablation}

Durch die Applikation von Radiofrequenzstromenergie wurden bei pädiatrischen Patienten Komplikationen mit einer Prävalenz von 3 bis 4,2 \% beobachtet (Brugada et al. 2013). Hauptsächlich handelte es sich hierbei um Koronaraffektionen, thrombembolische Ereignisse, Perforationen, Perikardergüsse oder iatrogen hervorgerufene Herzrhythmusstörungen. Bei den durch Radiofrequenzstromenergie induzierten Herzrhythmusstörungen ist in erster Linie der schrittmacherpflichtige, permanente AV-Block III ${ }^{\circ}$ zu nennen. Es wurden aber auch tachykarde Herzrhythmusstörungen im Langzeitverlauf nach RFA beschrieben (Koch et al. 2015, Brugada et al. 2013, Blomström-Lundqvist et al. 2003, Deisenhofer et al. 2010). Auch Todesfälle als Komplikation der Radiofrequenzablation von Substraten supraventrikulärer Tachykardien wurden beschrieben (Brugada et al. 2013). Mittlerweile ist allgemein akzeptiert, dass ein Körpergewicht von unter $15 \mathrm{~kg}$ zum Zeitpunkt der Ablation mit einem erhöhten Risiko für unerwünschte Nebenwirkungen bei der Radiofrequenzablation einhergeht (Schaffer 2000, Blaufox et al. 2004, Saul et al. 2016).

Hinsichtlich der Sicherheit insbesondere in Bezug auf das Auftreten von Schäden des spezifischen Reizleitungssystems sowie Schädigungen der Koronararterien gibt es Hinweise für einen Vorteil der Kryoenergieanwendung gegenüber der 
RFA (Kriebel et al. 2005, Aoyama et al. 2005, Kriebel et al. 2009, Schneider et al. 2009).

\subsection{Koronaraffektionen}

Die Koronaraffektion ist eine schwere Komplikation der Katheterablation und potentiell lebensbedrohlich. Die zur Elimination der Tachykardiesubstrate führenden Gewebeeffekte können auch (in Abhängigkeit von der Entfernung zur Energiequelle) die Wände der Koronararterien schädigen und so zu Lumeneinengungen und konsekutiver Myokardischämie führen. Auch reaktive Spasmen der Koronararterien oder Ödembildung in der Wand der Koronararterien können ohne morphologische Schädigung der Gefäßwand zu einer verminderten Myokardperfusion und konsekutiver Ischämie führen (siehe Abbildung 6).

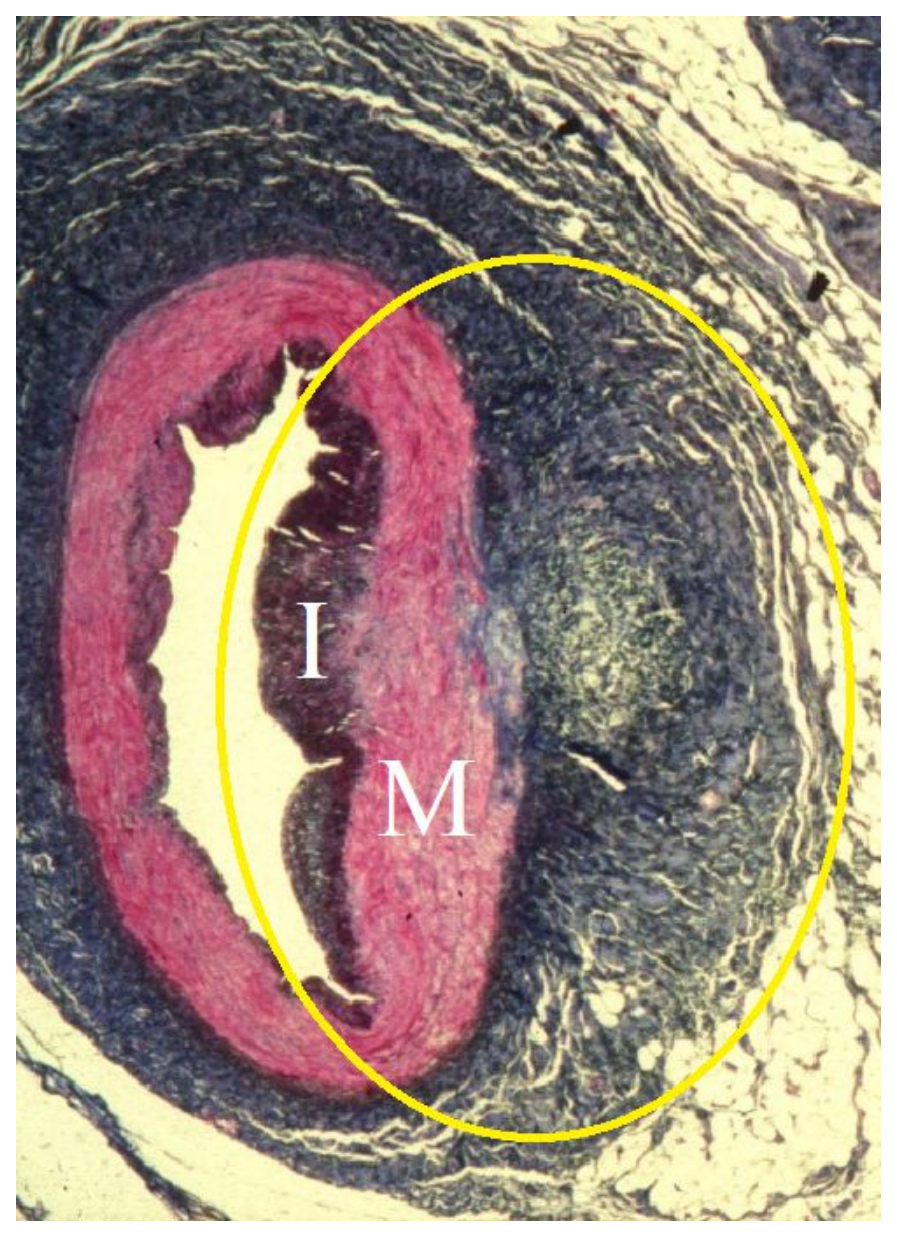

Abbildung 6: Stenose der rechten Koronararterie eines Schweines mit Lumeneinengung um 40\% 6 Monate nach RFA mit deutlicher Zellproliferation im Bereich der Intima (I) und Media auf der läsionszugewandten Seite (gelbe Markierung). (Paul et al. 1997, Mit freundlicher Genehmigung des Elsevier-Verlags)

Klinische Studienergebnisse zeigten, dass die Prävalenz von Koronaraffektionen nach Katheterablation bei Kindern 0,03 \% (Blaufox und Saul 2004, Kugler et al. 
1997) und bei Erwachsenen 0,06 bis 0,1 \% (Calkins et al. 1992, Scheinman und Huang 2000) betrug. Ebenso konnte gezeigt werden, dass es vor allem bei posteroseptal gelegenen akzessorischen Leitungsbahnen postinterventionell zu Lumenverengungen kommen kann (Schneider et al. 2009).

\subsection{0 latrogen hervorgerufene Herzrhythmusstörungen}

Bei kathetergestützten Eingriffen mit Radiofrequenzenergie am Herzen kann es auch zu Schädigungen des normalen Erregungsbildungs- und Leitungssystems des Herzens kommen. Daraus können dann bradykarde Herzrhythmusstörungen wie ein AV-Block oder eine Sinusknotendysfunktion resultieren. Das Risiko für das Auftreten von iatrogen erzeugten Rhythmusstörungen liegt bei 1 bis $2 \%$ (Brugada et al. 2013). Bei der Ablationstechnik mittels Kryoenergie konnte dieses Risiko durch das Kryomapping deutlich gesenkt und das Sicherheitsprofil erhöht werden. Das Kryomapping erlaubt eine reversible Läsionsformation und beugt somit permanenten Schädigungen des spezifischen Reizleitungssystems des Herzens vor. So wurde beispielsweise bisher bei der Anwendung von Kryoenergie kein permanenter AV-Block in der internationalen Literatur beschrieben (Brugada et al. 2013).

Neben bradykarden Herzrhythmusstörungen sind aber auch tachykarde Herzrhythmusstörungen infolge der Katheterablation einer supraventrikulären Tachykardie beschrieben (siehe Abbildung 7) (Koch et al. 2016). 


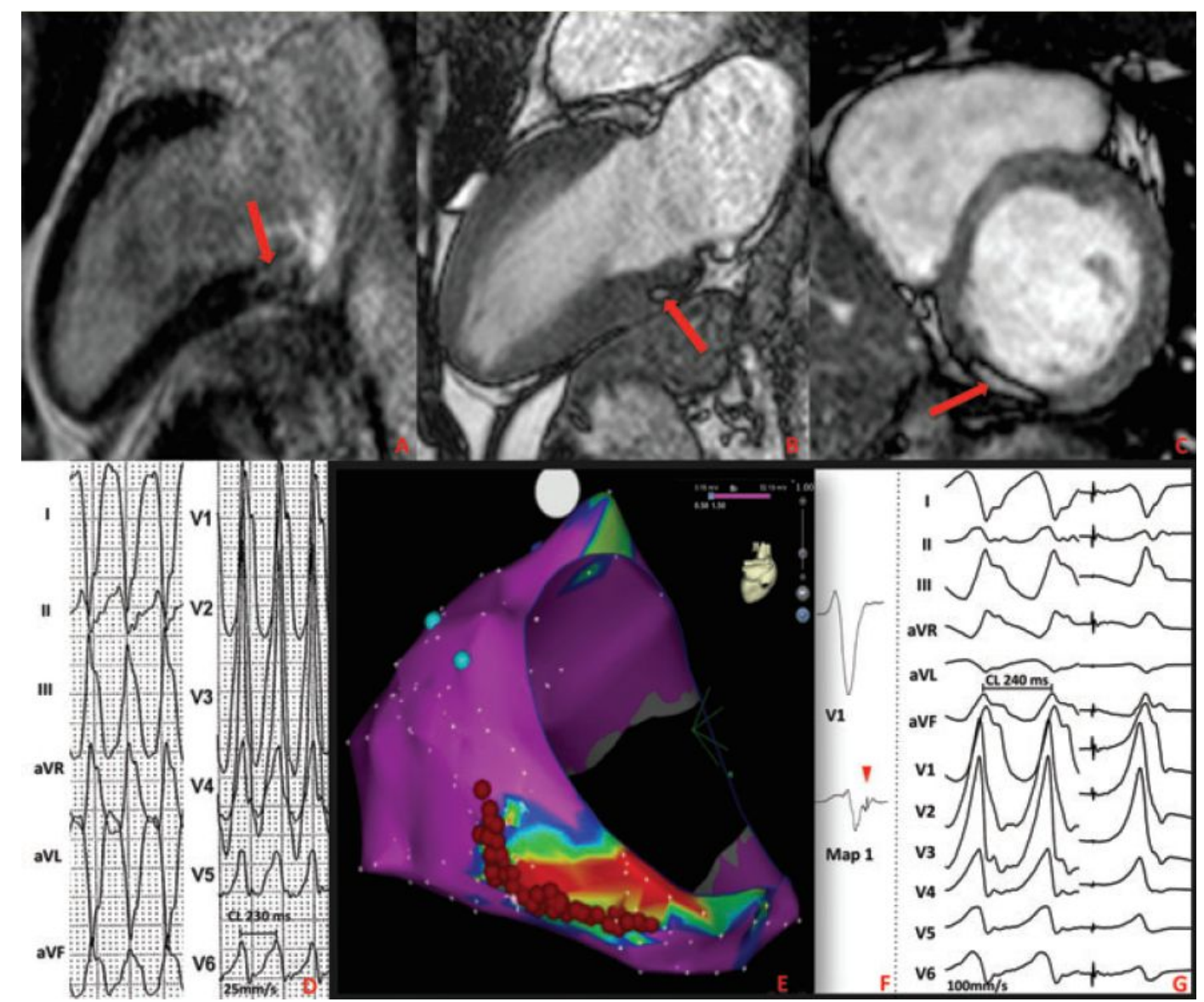

Abbildung 7: Diagnostik bei Patienten mit VT nach RFA einer linksseitig lokalisierten akzessorischen atrioventrikulären Leitungsbahn, hyperintenses Signal im linksventrikulären inferobasalem Segment (roter Pfeil Bild A), hyperintenses Signal umgeben von signalfreier Grenzzone übereinstimmend mit Fettgewebe in myokardialem Narbengewebe (roter Pfeil Bilder B und C), VT (Bild D), „low-voltage" Bereiche im Narbengebiet in der EPU (Bild E), Ablation und sistieren der VT (Bilder E-G) (Koch et al. 2016, Mit freundlicher Genehmigung der Oxford University Press).

\subsection{Fragestellung und Zielsetzung}

Die im Rahmen der vorliegenden Dissertationsarbeit durchgeführte Studie sollte die Frage beantworten, ob die Applikation zusätzlicher Kryoenergie Läsionen (drei konsekutive Anfrier-Auftau-Zyklen an einem Punkt) die Effektivität der Kryoenergieablation bei gleichbleibend hoher Sicherheit in Bezug auf Schädigungen des spezifischen Reizleitungssystems sowie der Koronararterien im unreifen Myokard erhöht. In der vorliegenden Studie wurden die Gewebeeffekte nach drei konsekutiven Anfrier-Auftauzyklen früh-postinterventionell nach 48 Stunden untersucht. Die Studie wurde unter der Annahme durchgeführt, dass bei der dreifachen Kryoenergieapplikation ein größeres Läsionsvolumen erzeugt werden kann als bei einfacher oder doppelter Kryoenergieapplikation, was zu einer gesteigerten dauerhaften Effektivität führen soll. Ebenfalls sollte untersucht werden, ob 
durch die größeren Läsionsvolumina die Rate der Komplikationen steigt. Besonderes Augenmerk wurde hierbei auf die Affektion von Koronararterien gelegt. Die Ergebnisse dieser Studie sollten weitere wichtige Beiträge zur Indikationsstellung und Risikoabwägung der Kryoenergieablationstechnik in der pädiatrischen Kardiologie leisten. 


\section{Material und Methoden}

Für die Durchführung der tierexperimentellen Studie lag eine Genehmigung des Niedersächsischen Landesamtes für Verbraucherschutz und Lebensmittelsicherheit unter dem Aktenzeichen 33.9-42502-04-13/1119 vor.

Um die Ergebnisse des Tierexperimentes auf den Menschen übertragen zu können, ist es wichtig vergleichbare Voraussetzungen zu schaffen. Aufgrund der starken Ähnlichkeit zwischen dem Herzen des Schweines und dem des Menschen, eignen sich Schweine besonders als Modelltiere in der Kardiologie. Von großer Bedeutung für die Übertragung der tierexperimentellen Ergebnisse auf den Menschen sind vor allem die Anordnung der Koronararterien, das nahezu identisch aufgebaute spezifische Reizleitungssystem und die Fähigkeit nach myokardialer Ischämie Kollateralen bilden zu können (Crick et al. 1998).

In der Versuchsreihe wurden insgesamt sieben Schweine vom Typ „Deutsche Landrasse“ untersucht. Zum Zeitpunkt der Katheterablation mit Kryoenergie hatten die Tiere ein Gewicht zwischen 15 - 20 kg und ein Alter von 6 - 8 Wochen. Das entspricht, auf den Menschen übertragen, einem Kleinkind. Die artgerechte Haltung erfolgte auf einem Versuchsgut im niedersächsischen Holtensen, bevor die Tiere drei bis fünf Tage vor Versuchsbeginn in die Zentrale Tierexperimentelle Einrichtung (ZTE) der Universitätsmedizin Göttingen transportiert wurden. Zu jedem Zeitpunkt der Studie war die Betreuung der Tiere durch einen erfahrenen Veterinärmediziner und Tierpfleger gegeben.

\subsection{Vorbereitung, Voruntersuchungen und Anästhesie}

Vor Beginn der Herzkatheteruntersuchung und zur Vorbereitung der Narkose erfolgte die Prämedikation der Tiere mit einer intramuskulären Applikation von 80 mg/kg Körpergewicht Azaperon (Strensil ®, Jansen-Cilag, Neuss, Deutschland). Nach Eintreten der gewünschten sedierenden Wirkung wurde die Vena auricularis an einem der beiden Ohren mit einer Venenverweilkanüle punktiert und somit ein venöser Zugang geschaffen. Über diesen Zugang erfolgte die intravenöse Einleitung der Narkose unter Einhaltung des folgenden Standardverfahrens: Zur Einleitung der Narkose erhielten die Tiere 0,1 mg/kg Körpergewicht Midazolam (Midazolam-Ratiopharm ${ }^{\circledR}$, Ratiopharm GmbH, Ulm, Deutschland) sowie $1 \mathrm{mg} / \mathrm{kg}$ Körpergewicht Ketamin (Ketamin-Ratiopharm, Ratiopharm 
$\mathrm{GmbH}$, Ulm, Deutschland). Die Aufrechterhaltung der Anästhesie erfolgte mittels Gasnarkose unter Verabreichung von Isofloran 2 \% (Forene $\AA$, Abbott $\mathrm{GmbH}$ und Co. KG, Wiesbaden, Deutschland) und zusätzlicher Gabe von Propofol (Propofol-Lipuro ${ }$, Braun Melsungen AG, Melsungen, Deutschland) in der Dosierung von $1 \mathrm{mg} / \mathrm{kg}$ Körpergewicht/Stunde. Nach erfolgreicher endotrachealer Intubation in Brust-Bauch-Lage wurden die Tiere an das Narkosegerät "Sulla19“ der Firma Dräger (Drägerwerk AG, Lübeck, Deutschland) angeschlossen. Der Narkoseverlauf wurde dokumentiert und die Vitalparameter kontinuierlich überwacht. Zum Monitoring der Vitalparameter wurden die transkutane Sauerstoffsättigung und der Kohlenstoffdioxidgehalt in der Exspirationsluft gemessen, sowie ein Extremitäten-Elektrokardiogramm abgeleitet. Die kontinuierliche invasive Blutdruckmessung erfolgte nach Punktion der A. femoralis und Platzierung der arteriellen Schleuse. Zur Anlage der Schleusen wurden die Schweine in Rückenlage verbracht und anschließend fixiert (siehe Abbildung 8). Unter sterilen Bedingungen wurden im Gebiet der geplanten Punktionsstellen im Inguinalbereich ca. $30 \mathrm{mg}$ des Lokalanästhetikums Lidocain (Xylocain, Astra Zeneca, Wendel, Deutschland) injiziert. Die Arteria und Vena femoralis wurden palpatorisch lokalisiert und dann mit einer Standard-Punktionsnadel (18 G, Cook Medical, Bloomington, USA) punktiert. Anschließend wurden in Seldinger-Technik jeweils eine 7-French-Schleuse (7 F) in die Gefäße eingelegt (Cordis, Roden, Niederlande). Nach Platzierung der Schleusen erfolgte die Antikoagulation mit unfraktioniertem Heparin (100 IE/kg Körpergewicht, Heparin-Natrium, Ratiopharm GmbH, Ulm, Deutschland) über die venöse Schleuse. Gleichermaßen erfolgte die einmalige prophylaktische i. v. Gabe von $50 \mathrm{mg} / \mathrm{kg}$ Körpergewicht des Antibiotikums Cephazolin (Cephazolin, Fresenius Kabi AG, Bad Homburg, Deutschland).

Zur selektiven angiographischen Beurteilung der rechten und linken Koronararterien wurde über die zuvor platzierte arterielle Schleuse ein 5-French-Koronarangiographiekatheter ( $5 \mathrm{~F}$ Amplatz re/li oder $5 \mathrm{~F}$ Judkins li, Cordis, Roden, Niederlande) bis in die Aortenwurzel vorgeschoben und die Koronargefäße durch selektive Kontrastmittelgabe dargestellt. Die selektiven Angiographien der Koronararterien wurden hinsichtlich Stenosen, arteriosklerotischer Plaqueformationen oder sonstiger pathologischer Anomalien untersucht. Die Röntgen-Durchleuchtung erfolgte mit einem mobilen Röntgengerät (BV Pulsera, Firma Phillips, Amsterdam, Niederlande) in den Standardprojektionsebenen $30^{\circ}$ RAO (rechts- 
anterior-oblique) und $60^{\circ}$ LAO (links-anterior-oblique). Die Aufnahmen wurden für die Auswertung digital gespeichert. Die Punktion der inguinalen Gefäße, die Koronarangiographien sowie die intrakoronare Ultraschalluntersuchung (s. u.) und die Induktion der Kryoenergie-Läsionen wurden von einem in der Durchführung von Herzkatheteruntersuchungen und Katheterablationen bei Kindern und Jugendlichen erfahrenen Kinderkardiologen durchgeführt.

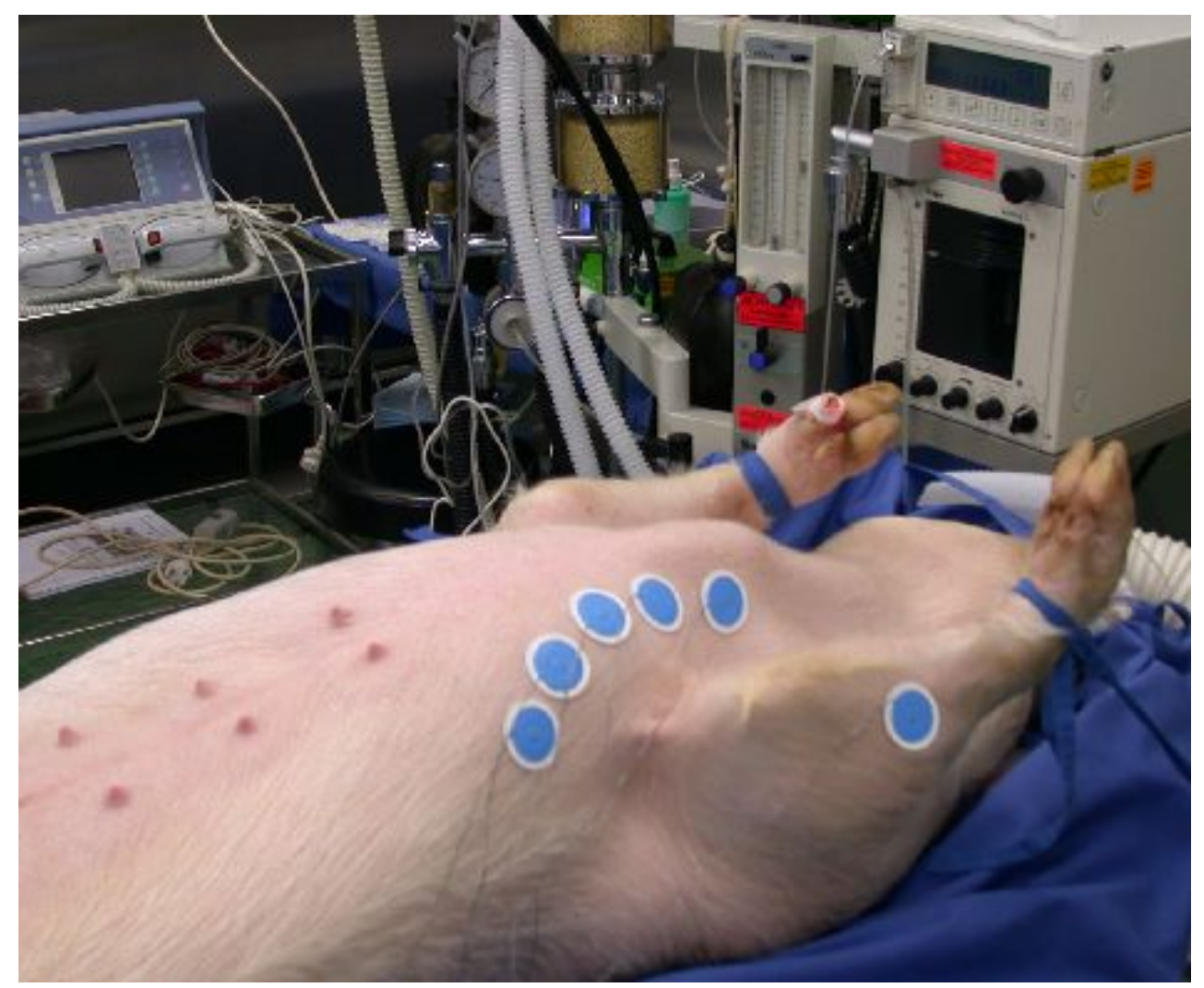

Abbildung 8: Intubiertes und in Rückenlage fixiertes Schwein. Auf dem Brustkorb EKG-Elektroden und an der rechten Vorderpfote ein SpO2-Sensor

\subsection{Kryoablation}

Nach Durchführung der Koronarangiographien wurde ein Kryoenergie-Ablationskatheter (7 F FreezorXtra®, 6 mm Spitze, Medtronic, Minneapolis, USA) über die venöse Schleuse bis zum Trikuspidalklappenanulus vorgeschoben. Der Ablationskatheter wurde an eine CryoCath $®$-Konsole (Medtronic, Minneapolis, USA) angeschlossen. Die korrekte Positionierung des Ablationskatheters wurde zum einen über die Analyse der bipolaren Elektrogramme, welche über das distale Elektrodenpaar des Ablationskatheters abgeleitet wurden (Amplitude atrialer und ventrikulärer Elektrogramme) sowie fluoroskopisch bestimmt. Es wurden jeweils 
Kryoenergieläsionen am atrialen und am ventrikulären Rand des Trikuspidalklappenanulus induziert. Mit einer Temperatur von -70 bis $-80^{\circ} \mathrm{C}$ und über die Dauer von vier Minuten wurde die Kryoenergie über die Spitze des Katheters auf das Myokard übertragen. Nach Beendigung der Kryoenergieapplikation erfolgten an der gleichen Stelle nach einer Auftauzeit von einer Minute jeweils zwei weitere Kryoenergieapplikationen, so dass Läsionen durch einen Anfrier-Auftau-AnfrierAuftau-Anfrier-Auftau-Zyklus induziert wurden. Um die exakt identische Positionierung des Ablationskatheters an einer Stelle für die darauffolgenden Kryoenergieabgaben sicherzustellen, wurde ein elektroanatomisches Navigationssystem (LocaLisa ${ }^{\circ}$, Medtronic, Minneapolis, USA) verwendet. Dieses System erlaubt die Repositionierung eines Katheters an einer identischen Stelle mit einer Punktgenauigkeit von 1,4 $\pm 1,1 \mathrm{~mm}$ (Wittkampf et al. 1999). Nachdem jeweils drei Läsionen am atrialen und ventrikulären Aspekt der Trikuspidalklappe induziert waren, erfolgte die retrograde, transaortale Platzierung des Ablationskatheters am Mitralklappenanulus. Am Mitralklappenanulus wurden ebenfalls je drei Kryoenergieläsionen am atrialen und am ventrikulären Aspekt der Mitralklappe durch Wiederholung von jeweils drei Anfrier-Auftau-Zyklen pro Läsion induziert.

Nachdem alle Kryoenergie-Läsionen in den Tieren induziert worden waren, erfolgte erneut eine selektive Angiographie beider Koronararterien, welche insbesondere im Hinblick auf neu aufgetretene Stenosen oder Wandunregelmäßigkeiten nach Kryoenergieapplikation untersucht wurden. Nach Abschluss der invasiven Maßnahmen wurden die inguinal liegenden Schleusen entfernt und Druckverbände angelegt. Die Narkose wurde ausgeleitet und nach sichergestellter Spontanatmung und bei ausreichenden Schutzreflexen erfolgte die Extubation der Schweine. Zur post-interventionellen Überwachung wurden die Schweine zurück in die Ställe der ZTE der Universitätsmedizin Göttingen gebracht. Um die Bildung von Adhäsionsthromben zu vermeiden, erhielten die Tiere für die folgenden zwei Tage täglich 2 - 3 mg/kg Acetylsalicylsäure per os.

\subsection{Nachuntersuchung $48 \mathrm{~h}$ nach dreifacher Kryoenergieappli- kation}

48 Stunden nach der dreifachen Kryoenergieapplikation wurden die Schweine einer erneuten selektiven Angiographie beider Koronararterien unterzogen. Die 
Untersuchung erfolgte ebenfalls in Intubationsnarkose und wurde durch einen erfahrenen Veterinärmediziner begleitet. Die Vorgehensweise entsprach dabei den angiographischen Voruntersuchungen (siehe oben), mit dem Unterschied, dass dieses Mal nur eine arterielle Schleuse $(7 \mathrm{~F})$ gelegt wurde. Anschließend erfolgte dann eine intrakoronare Ultraschalluntersuchung (ICUS) der rechten und linken Koronararterien. Mittels dieses Verfahrens war es möglich, die Wandstruktur der Koronararterien auf pathologische Veränderungen, Plaqueformationen, Adhäsionsthromben oder andere stenosierende Prozesse zu untersuchen. Hierfür wurde über einen 0,014 Inch starken Führungsdraht (Hi-Torque, Balance Middleweight, Abbott, Diegem, Belgien) ein 2,9 French starker Eagle Eye Platinum (B) Ultraschallkatheter (Volcano Europe, Zaventem, Belgien) bis in die distale rechte bzw. linke Koronararterie vorgeschoben. Die Ultraschalluntersuchung erfolgte mit einer Frequenz von $20 \mathrm{MHz}$. Der Ultraschallkatheter wurde möglichst weit distal in das Koronargefäß vorgebracht und unter fluoroskopischer Kontrolle langsam manuell bis zum Erreichen des Gefäßostiums retrahiert. Die erhobenen Untersuchungsdaten wurden digital gespeichert (Volcano $\mathrm{s} 5 \mathrm{i}^{\mathrm{TM}}$ imaging system, Volcano, San Diego, USA) und waren somit auch nachträglich noch offline für weitere Analysen zugänglich.

\subsection{Herzentnahme}

Nach Abschluss der ICUS-Untersuchung erfolgte die Euthanasie der Schweine. Hierfür wurde den Tieren eine Überdosis Pentobarbital durch den Veterinärmediziner intravenös appliziert. Unmittelbar nach sicherem Eintreten des Todes erfolgte die Entnahme der Herzen. Mittels einer medianen Sternotomie wurde der Brustkorb eröffnet und das Perikard aufgetrennt. Das Herz und die umliegenden Strukturen wurden freipräpariert und dargestellt. Die großen Gefäße wie die Aorta, die Hohlvenen und die Pulmonalgefäße wurden durch Ligaturen abgebunden und konnten schließlich abgesetzt werden. Losgelöst konnte das Herz nun entnommen und zunächst in physiologischer Kochsalzlösung gewaschen werden.

\subsection{Makroskopische Untersuchung}

Nach der Entnahme der Herzen wurden diese gewogen und anschließend schonend eröffnet. Im Inneren von Atrium und Ventrikel wurde nun im Bereich der Mitral- und Trikuspidalklappe nach makroskopisch sichtbaren Läsionen gesucht 
(siehe Abbildung 2). Jede Läsion erhielt unmittelbar nach Identifizierung eine eigene Nummer. Die Läsionen wurden fotografiert (Nikon D5000, Digitalkamera) und mit einem Lineal in ihrer Ausdehnung makroskopisch vermessen. Die Läsionsnummer, die Lokalisation der Läsion sowie die Ergebnisse der makroskopischen Vermessung und sonstige Auffälligkeiten wurden dokumentiert.

\subsection{Histologische Aufarbeitung}

Die Arbeitsschritte der histologischen Aufarbeitung fanden im Forschungslabor der Klinik für Pädiatrische Kardiologie und Intensivmedizin der Universitätsmedizin Göttingen statt. Nach der Identifizierung und makroskopischen Untersuchung und Vermessung der Läsionen wurden die entsprechenden Areale in großzügigen Gewebsblöcken mit dazugehörigem AV-Klappenanulus und Koronargefäß herausgeschnitten und in 10 \%iger Formalinlösung (Rotiß Histofix, Carl RothGmbH und Co. KG, Karlsruhe, Deutschland) fixiert. Anschließend wurden die fixierten Gewebsblöcke unter Verwendung eines Gewebeeinbettautomaten (Shandon-Citadel $1000 \AA$, Shandon $\mathrm{GmbH}$, Frankfurt am Main, Deutschland) in Paraffin eingebettet. Dieses erfolgte nach nachfolgendem Schema: 
Tabelle 1: Arbeitsschritte zur Fixierung mit aufsteigender Alkoholreihe

\begin{tabular}{|c|c|c|}
\hline Schritt & Lösungmittel & Zeit \\
\hline 1 & $\mathrm{H}_{2} \mathrm{O}$ bzw. Ethanol $60 \%$ & 180 Minuten \\
\hline 2 & Ethanol $70 \%$ & 60 Minuten \\
\hline 3 & Ethanol $80 \%$ & 60 Minuten \\
\hline 4 & Ethanol $96 \%$ & 60 Minuten \\
\hline 5 & Ethanol $100 \%$ & 60 Minuten \\
\hline 6 & Ethanol $100 \%$ & 60 Minuten \\
\hline 7 & Ethanol $100 \%$ & 60 Minuten \\
\hline 8 & Xylol & 90 Minuten \\
\hline 9 & Xylol & 90 Minuten \\
\hline 10 & Paraffin & 60 Minuten \\
\hline 11 & Paraffin & 60 Minuten \\
\hline 12 & Paraffin & 180 Minuten \\
\hline
\end{tabular}

Paraffin besitzt die Eigenschaft, sich bei höheren Temperaturen auszudehnen, was sich negativ auf die Schneidefähigkeit auswirken kann. Um für den anschließenden Schneidevorgang eine bessere Stabilität und Festigkeit des fixierten und eingebetteten Gewebeblockes gewährleisten zu können, wurden diese zunächst tiefgekühlt. Nach Aushärtung der würfelförmigen Blöcke konnten diese mit Hilfe eines Rotationsmikrotoms (RM2165, Leica Microsystems $\mathrm{GmbH}$, Wetzlar, Deutschland) in Schnitte mit einer Schnittdicke von fünf Mikrometern geschnitten werden. Daraufhin wurden die Schnitte auf einen Objektträger (Superfrost+ Objektträger, Gerhard Menzel GmbH, Braunschweig, Deutschland) aufgetragen. Jeder Block und jeder Schnitt erhielt ebenfalls eine fortlaufende Nummer, die auf 
die Label-Seite des Objektträgers aufgetragen wurde. Somit war eine Zuordnung zum Tier, zur Läsion, zum Block und zur Schnittfolge gewährleistet.

\subsubsection{Histologische Färbungen}

Um eine mikroskopische Beurteilung der Präparate vornehmen zu können, wurden verschiedene Färbungen angefertigt. Alle 200 Mikrometer erfolgten eine Standard Hämatoxylin-Eosin-Färbung sowie eine Elastika-van-Gieson-Färbung. Die speziellen immunhistochemischen Desmin-, Fibrin- und von-Willebrand-Färbungen wurden nach jedem zehnten Schnitt angefertigt. Diese dienten einer besseren Gewebsdifferenzierung zwischen Muskulatur und Bindegewebe beziehungsweise zwischen geschädigtem und unversehrtem Gewebe.

\subsubsection{Färbetechniken}

Zunächst erfolgte die Rehydrierung der Gewebeschnitte, indem die Präparate eine absteigende Alkoholreihe durchliefen. Dieser Arbeitsschritt wurde für alle Färbemethoden identisch durchgeführt.

Tabelle 2: Arbeitsschritte zur Rehydrierung mit absteigender Alkoholreihe

\begin{tabular}{|c|c|c|}
\hline Wiederholungen & Lösungsmittel & Zeit \\
\hline 3 & Xylol & 8 Minuten \\
\hline 2 & Alkohol $100 \%$ & 5 Minuten \\
\hline 1 & Alkohol $96 \%$ & 5 Minuten \\
\hline 1 & Alkohol $70 \%$ & 5 Minuten \\
\hline 1 & Alkohol $50 \%$ & 5 Minuten \\
\hline 1 & Alkohol $30 \%$ & 5 Minuten \\
\hline 1 & Entionisiertes $\mathrm{H}_{2} \mathrm{O}$ & 5 Minuten \\
\hline
\end{tabular}

Nachfolgend sind die charakteristischen Arbeitsschritte der einzelnen Färbetechniken aufgeführt, die sich dem oben angegebenen Schritt anschließen. 


\subsubsection{Hämatoxylin-Eosin-Färbung}

Tabelle 3: Arbeitsschritte zur Anfertigung der Hämatoxylin-Eosin-Färbung

\begin{tabular}{|c|c|c|}
\hline Wiederholungen & Lösungsmittel & Zeit \\
\hline 3 & Meyers Hämalaunlösung & 3 Minuten \\
\hline 1 & Fließendes Leitungswasser & 6 Minuten \\
\hline 1 & Entionisiertes $\mathrm{H}_{2} \mathrm{O}$ & 2 Minuten \\
\hline 2 & Eosinlösung $5 \%$ & 2 Minuten \\
\hline 1 & Entionisiertes $\mathrm{H}_{2} \mathrm{O}$ & spülen \\
\hline
\end{tabular}

Meyers Hämalaunlösung (Carl Roth $\mathrm{GmbH} \&$ Co. KG, Karlsruhe, Deutschland) Eosinlösung $5 \%$ (Carl Roth $\mathrm{GmbH} \&$ Co. KG, Karlsruhe, Deutschland)

\subsubsection{Elastika-van-Gieson-Färbung}

Tabelle 4: Arbeitsschritte zur Anfertigung der Elastika-van-Gieson-Färbung

\begin{tabular}{|c|c|c|}
\hline Wiederholungen & Lösungsmittel & Zeit \\
\hline 1 & Elastin nach Weigert & 10 Minuten \\
\hline 1 & Fließendes Leitungswasser & 1 Minute \\
\hline 1 & Weigert A \& B 1:1 & 5 Minuten \\
\hline 1 & Fließendes Leitungswasser & 1 Minute \\
\hline 1 & Pikrofuchsin-Lösung & 2 Minuten \\
\hline
\end{tabular}

Elastin nach Weigert (Firma Merck, Darmstadt, Deutschland) Weigert A \& B 1:1 (Firma Merck, Darmstadt, Deutschland) 


\subsubsection{Immunhistochemische Färbung}

Um die Präparate den immunhistochemischen Farbstoffen zugänglich zu machen, musste das Paraffin zunächst aus dem Gewebe entfernt werden. Die sogenannte Entplastung lief nach nachfolgendem Schema ab:

Tabelle 5: Arbeitsschritte der Entplastung

\begin{tabular}{|c|c|c|}
\hline Wiederholungen & Lösungsmittel & Zeit \\
\hline 3 & Xylol & 20 Minuten \\
\hline 1 & 2-Methoxyethylacetat & 20 Minuten \\
\hline 2 & Aceton reinst & 5 Minuten \\
\hline 1 & Entionisiertes $\mathrm{H}_{2} \mathrm{O}$ & 5 Minuten \\
\hline
\end{tabular}

Das Prinzip immunhistochemischer Färbungen basiert auf der Bildung von Antigen-Antikörper-Komplexen. Um bestimmte Gewebskomponenten sichtbar zu machen, werden Antigene dieser Strukturen durch die Zugabe von unkonjugierten Primärantikörpern, die sich gegen die Gewebsantigene richten, markiert. Die Detektion dieser Antigen-Antikörper-Komplexe erfolgt durch die Zugabe von Sekundärantikörpern, die sich gegen die Primärantikörper richten. Da sich die Färbeschritte der immunhistochemischen Färbungen lediglich in der Vorbehandlung, d. h. in der Demaskierung der Gewebsantigene, und der verwendeten Antikörper unterscheiden, erfolgt zunächst eine tabellarische Aufführung des allgemeinen Färbeprotokolls und anschließend die Erläuterung der Unterschiede in Vorbehandlung und Antikörpern. 
Tabelle 6: Arbeitsschritte des allgemeinen immunhistochemischen Färbeprotokolls

\begin{tabular}{|c|c|}
\hline Vorbehandlung & Färbungsspezifisch (s.u.) \\
\hline Waschen & $5 \times 2$ Minuten mit TTBS \\
\hline Blocken der endogen Peroxidase & DAKO Peroxidase Blocking Reagent S001 \\
\hline Inkubation & 12 Minuten bei Raumtemperatur \\
\hline Waschen & $5 \times 2$ Minuten mit TTBS \\
\hline Inkubation mit AK-Diluent & 30 Minuten \\
\hline 1. Antikörper & Siehe unten \\
\hline Inkubation & 60 Minuten bei Raumtemperatur \\
\hline Waschen & $5 \times 2$ Minuten mit TTBS \\
\hline 2. Antikörper & Siehe unten \\
\hline Inkubation & 60 Minuten bei Raumtemperatur \\
\hline Waschen & $5 \times 2$ Minuten mit TTBS \\
\hline Inkubation & 30 Minuten bei Raumtemperatur \\
\hline Substrat-Chromogensystem & $\begin{array}{l}\text { DAB frisch ansetzen: pro } 1 \mathrm{ml} \text { Substrat } \\
\text { 1Tropfen Liquid DAB Chromogen } \\
\text { Inkubation: 5-30 Minuten unter Mikroskopkon- } \\
\text { trolle }\end{array}$ \\
\hline Waschen & $\begin{array}{l}\text { 4x1 Minute mit Aqua destillata } \\
\text { 1x5Minuten mit Aqua destillata }\end{array}$ \\
\hline Gegenfärben & $\begin{array}{l}15 \text { Sekunden- } 1 \text { Minute mit Hämalaun } \\
\text { 7Minuten unter fließendem Leitungswasser }\end{array}$ \\
\hline
\end{tabular}




\subsubsection{Desmin-Färbung}

Die Vorbehandlung der Desmin-Färbung bestand aus der Hinzugabe eines Puffers (Target Retrieval Puffer high $\mathrm{pH}$ ) in einen Dampfkochtopf bei $90^{\circ} \mathrm{C}$ über eine Inkubationszeit von 20 Minuten. Als Primär-Antikörper kam ein Maus-Antikörper

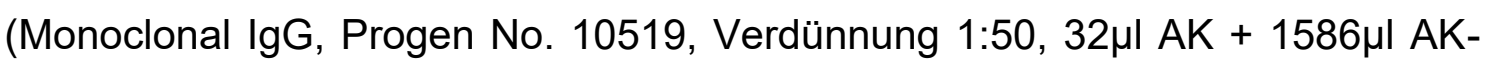
Diluent) zur Verwendung. Dieser musste bei $4{ }^{\circ} \mathrm{C}$ über Nacht inkubieren. Die Hinzugabe des Sekundär-Antikörpers (Rabbit-Anti-Mouse Immunglobulin DAKO P0260, Verdünnung 1:100,16 $\mu \mathrm{AK}+1584 \mu \mathrm{l}$ AK-Diluent) erfolgte mit einer Inkubationszeit von 60 Minuten unter Raumtemperatur.

\subsubsection{Fibrin-Färbung}

Hier bestand die Vorbehandlung in der Hinzugabe eines Citrat-Puffers $(\mathrm{pH} 6$, DAKO S2031) ebenfalls in einen Dampfkochtopf bei $90{ }^{\circ} \mathrm{C}$ und einer Inkubationszeit von 40 Minuten. Der Primärantikörper war ein Maus-Antikörper (Monoclonal Mouse-Anti-Human Fibrin ß-chain, American diagnostica Inc. ADI No. 350, Verdünnung 1:500) und als Sekundärantikörper wurde ein gegen Maus-Immunglobuline gerichteter Kaninchen-Antikörper (Rabbit-Anti-Mouse Immunglobulin DAKO P0260, Verdünnung 1:100) verwendet.

\subsubsection{Von-Willebrand-Färbung}

Zur Demaskierung der Gewebsantigene wurde auch bei dieser Färbung ein $\mathrm{Ci}$ trat-Puffer (pH 6, DAKO S2031) im Rahmen der Vorbehandlung verwendet. Dieser wurde ebenfalls in einem Dampfkochtopf bei $90^{\circ} \mathrm{C}$ über 40 Minuten inkubiert. Hier wurde zunächst ein Kaninchen-Antikörper (Anti-Human von-Willebrand-Faktor DAKO A0082, Verdünnung 1:400, $1 \mu \mathrm{IK}+399 \mu \mathrm{l}$ AK-Diluent) als Primärantikörper eingesetzt, bevor der Schweine-Antikörper (Swine-Anti-Rabbit Immunglo-

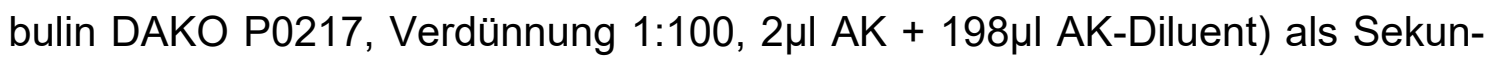
därantiköper Verwendung fand.

Nachdem alle Präparate mit ihrer jeweiligen Färbung gefärbt worden waren, schloss sich für jedes der Schritt der erneuten Dehydrierung an. Dieser wurde wie folgt durchgeführt: 
Tabelle 7: Arbeitsschritte der Dehydrierung nach histologischer Färbung

\begin{tabular}{|c|c|c|}
\hline Wiederholungen & Lösungsmittel & Zeit \\
\hline 1 & Alkohol $96 \%$ & 1 Minute \\
\hline 2 & Alkohol $100 \%$ & 2 Minuten \\
\hline 1 & Xylol & 3 Minuten \\
\hline
\end{tabular}

Abschließend erfolgte die Eindeckung mit Entellan $®$ (Firma Merck, Darmstadt, Deutschland). Die gefärbten Schnitte wurden nun nach der Schnittfolge geordnet in histologische Aufbewahrungskästen einsortiert und bis zur mikroskopischen Untersuchung im Forschungslabor der Klinik für Pädiatrische Kardiologie und Intensivmedizin der Universitätsmedizin Göttingen aufbewahrt.

\subsection{Mikroskopische Untersuchung}

Zur mikroskopischen Untersuchung der Schnittpräparate wurde ein virtuelles Mikroskopie-System der Firma Olympus (Olympus Life Science Europe GmbH, Hamburg, Deutschland) benutzt. Dieses System bestand aus einem Mikroskop (Typ BX51), einer Kamera (Typ 1376x1032 Pixel dotSlide Camera) und einer Objektträgeraufbewahrungseinheit. Das Mikroskop war mit Objektiven für 10-, 20- und 40-facher Vergrößerung ausgestattet. Die Aufbewahrungseinheit bot Platz für bis zu 50 Objektträger und war unterteilt in fünf Untereinheiten. Während die Einlage der Schnittpräparate manuell durch den Untersucher erfolgte, liefen der Scann- und Einlesevorgang automatisch ab. Ermöglicht wurde dieser Automatismus durch eine zwischengeschaltete Kontrollbox (BX-REMCB). Als Software diente das Programm „DotSlide 2.1@“ (DP-Soft Archivierungssoftware F DP 10, Olympus, Hamburg, Deutschland), an das die eingescannten Ausschnitte übermittelt wurden. In einem ersten Scanvorgang mit 20-facher Vergrößerung konnte der gewünschte Untersuchungsbereich eingegrenzt werden. Anschließend fotografierte die Kamera mit 40-facher Vergrößerung den vorgegebenen Bereich automatisch in Bilderfolgen ab und übermittelte die Bilder an das Computerprogramm. Die Software setzte die Einzelbilder nun wieder zu einem 
Ganzen zusammen, verarbeitete sie zu einer Datei und speicherte sie in einer Online-Datenbank.

\subsection{Flächenberechnung}

Die digitale Vermessung der eingescannten Präparate erfolgte unter Verwendung des Programmes "DotSlide ${ }^{\circ}$ (DP-Soft Archivierungssoftware F DP 10, Olympus, Hamburg, Deutschland). Die eingescannten Schnitte wurden vom Untersucher visuell beurteilt, anschließend wurden die Läsionen manuell und individuell in jedem Schnitt umfahren. Die Größe der umfahrenen Fläche entsprach dann der Läsionsfläche in dem untersuchten Gewebeanschnitt und wurde von der Software automatisch berechnet.

\subsection{Volumenberechnung}

Die durch DotSlide® ermittelten Werte wurden in Excel-Tabellen (Microsoft, Redmond, USA) exportiert. Zur Berechnung des Volumens der Läsionen wurde die Formel

Läsionsvolumen $=$ Grundfläche $\mathrm{x}$ Höhe

verwendet. Die Grundfläche entsprach hierbei der zuvor durch DotSlide® ermittelten Fläche. Für die Höhe wurde der Wert von 200 Mikrometern in die Formel eingesetzt, da die Schnitte zwar im Abstand von fünf Mikrometern angefertigt wurden, aber nur jeder 40. Schnitt aus Praktikabilitätsgründen planimetrisch vermessen wurde (40 x 5 Mikrometer = 200 Mikrometer $)$. Die Gesamtvolumina der Läsionen ließen sich anschließend nach der Formel: $\sum_{i}=\left(\right.$ Fläche $_{i}{ }^{*}$ Höhe $_{i}$ ) berechnen, wobei i die Anzahl der Schnitte pro Läsion darstellte.

\subsection{Statistische Auswertung}

Die statistische Auswertung der Ergebnisse wurde mit der Software SPSS (Version 22.0, IBM, Somers, NY, USA) durchgeführt. Sofern es nicht anders beschrieben ist, werden die Daten als Mittelwert \pm der Standardabweichung angegeben. Zur Bewertung der Ergebnisse konnten Vergleichsdaten von vorangegangenen Versuchsreihen mit doppelter Kryoenergieablation (Schneider et al. 2013) herangezogen werden. Zum Vergleich der Läsionsvolumina wurde ein ungepaarter t-Test durchgeführt. 
Der Vergleich zwei - und dreifacher Kryoenergieläsionen in der gleichen Versuchsreihe hätte eine höhere Anzahl von Versuchstieren erforderlich gemacht. Dies war von der zuständigen Behörde nicht genehmigt worden. Daher erfolgte der Vergleich der in der vorliegenden Arbeit induzierten Läsionen mit den Läsionen einer historischen Kohorte. Da die in der historischen Kohorte induzierten Läsionen aber am gleichen Modell und mit Ausnahme der Häufigkeit der Kryoenergieläsionen auch unter Verwendung des gleichen Versuchsprotokolls und eines identischen Ablationskatheters induziert worden waren, bestanden keine Bedenken gegen die Verwendung der historischen Daten als Vergleichsgruppe.

Bei einem vorgegebenen Signifikanzniveau von $\alpha=5 \%$ galt, dass bei einem p-Wert kleiner als 0,05 die Nullhypothese verworfen werden mußte und das Ergebnis als signifikant galt. 


\section{Ergebnisse}

\subsection{Herzrhythmusstörungen}

Bei keinem der Tiere traten während der Kryoenergieapplikation oder der folgenden Nachuntersuchungen eine Auffälligkeit im Oberflächen-EKG im Sinne tachykarder oder bradykarder Arrhythmien auf. Insbesondere kam es bei keinem der Versuchstiere zum Auftreten eines höhergradigen AV-Blocks.

\subsection{Ergebnisse der bildgebenden Diagnostik}

\subsubsection{Selektive Koronarangiographie}

Die Analyse der selektiven Koronarangiographien der linken und rechten Koronararterien, die unmittelbar vor Kryoenergieapplikation und 48 Stunden danach durchgeführt wurden, ergab keinen Anhalt für pathologische Prozesse. Es ließen sich keine Gefäßstenosen, Gefäßverschlüsse oder Wandunregelmäßigkeiten dokumentieren (s. Abbildung 9). 


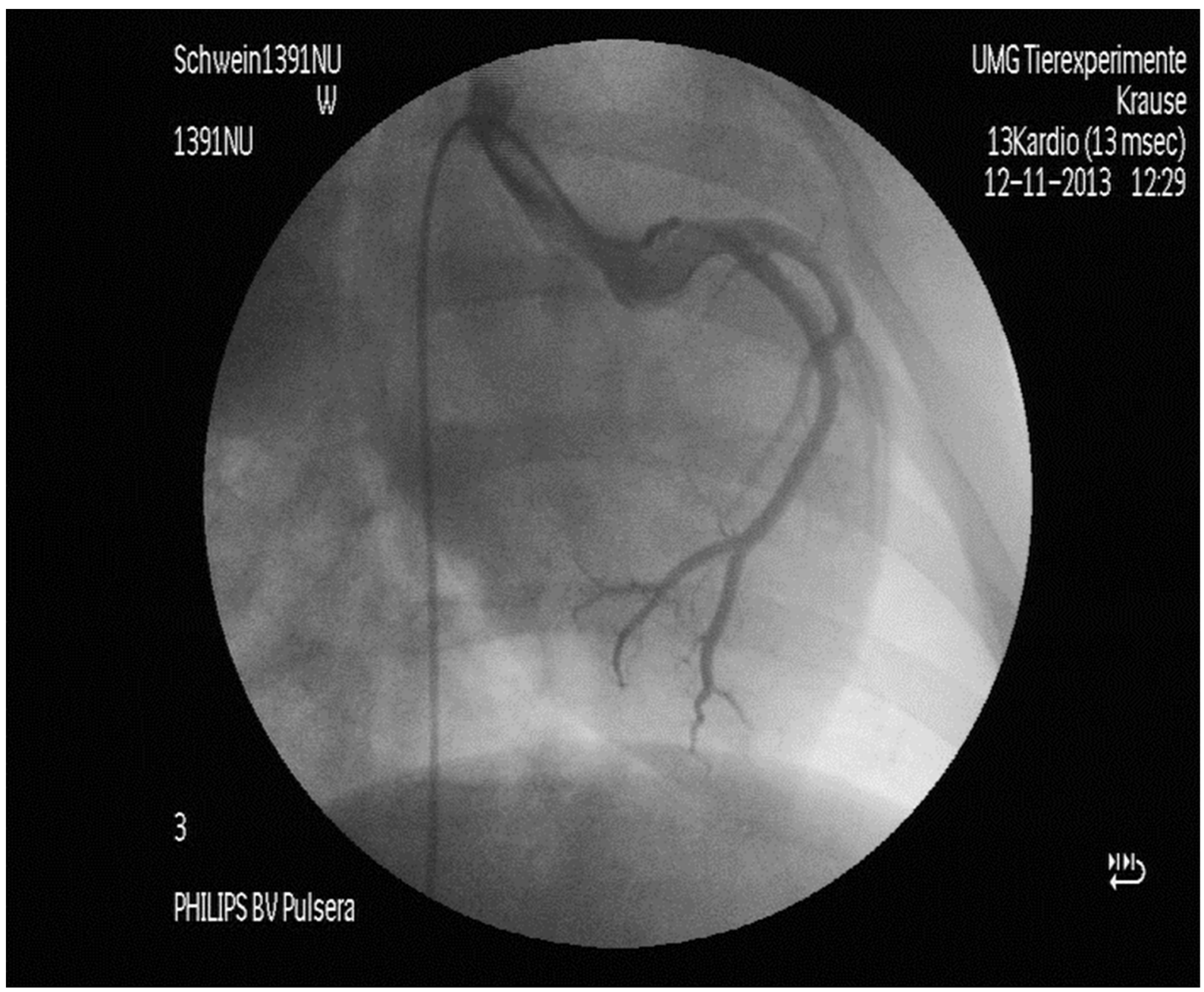

Abbildung 9: Koronarangiographie der LCA in LAO 60 eines der Testtiere $48 \mathrm{~h}$ nach Kryoenergieablation

\subsubsection{Intrakoronare Ultraschalluntersuchung (ICUS)}

Im Rahmen der Nachuntersuchung 48 Stunden nach Kryoenergieapplikation, wurde eine intrakoronare Ultraschalluntersuchung bei den Schweinen durchgeführt (s. Abbildung 10). Auch hierbei ließen sich keine Stenosen der Gefäße dokumentieren. Auch andere Zeichen, die hinweisend für eine Affektion der Koronararterien wären, wie Intimaproliferation, Plaques oder Thromben, waren mittels ICUS nicht nachweisbar. 


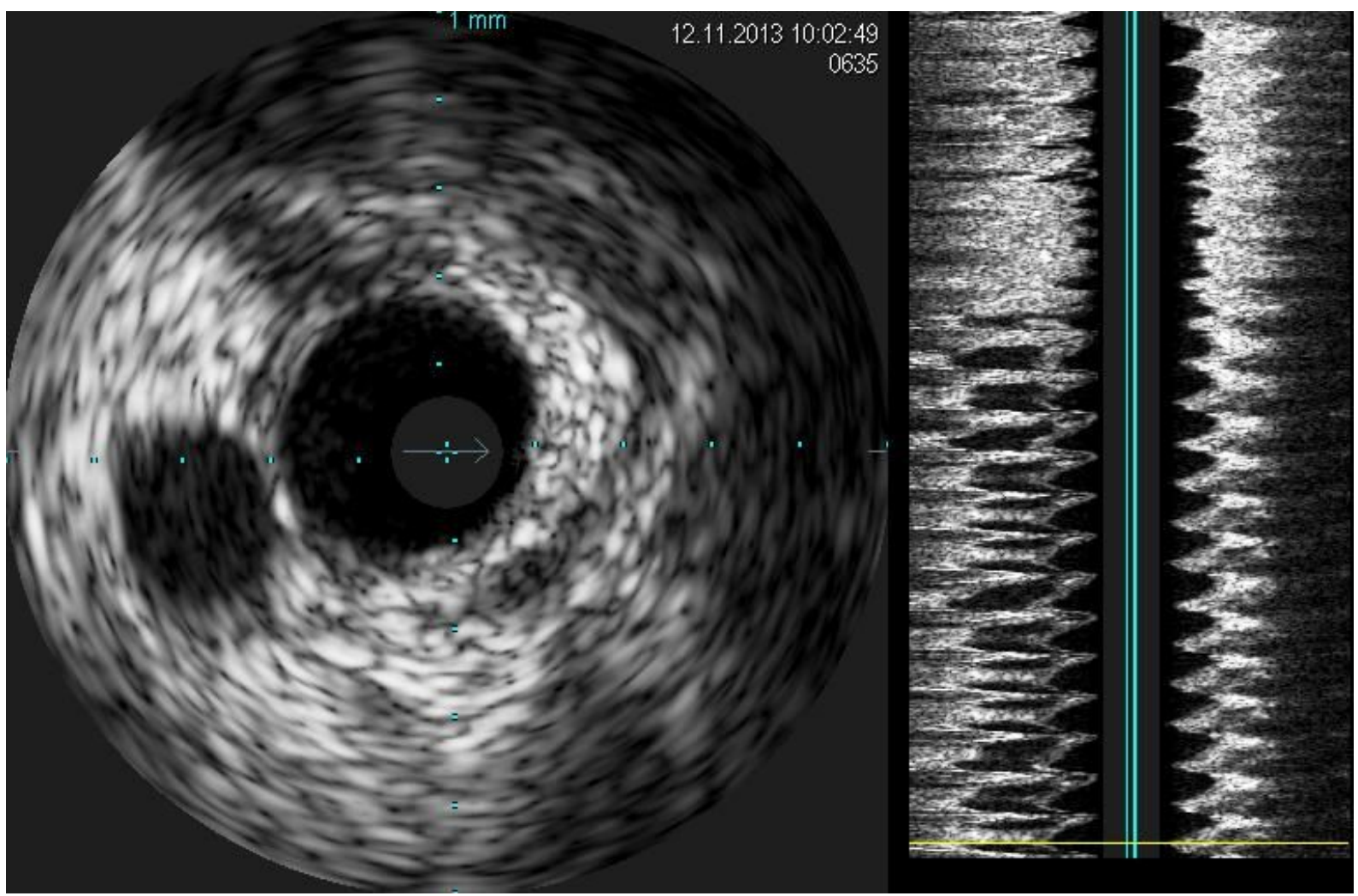

Abbildung 10: ICUS des Ramus inteventricularis anterior der linken Koronararterie eines der Testtiere 48 h nach Kryoenergieablation. Stenosen oder Plaques sind im Lumen des Gefäßes nach Kryoenergieapplikation nicht zu erkennen. Die linke Bildseite zeigt einen ostiumnahen repräsentativen Gefäßquerschnitt, die rechte Bildseite den Katheterrückzug (oben: distal, unten proximal).

\subsection{Makroskopische Ansicht der Läsionen}

48 Stunden nach dreifacher Kryoenergieapplikation konnten 30 Kryoenergieläsionen identifiziert und ausgewertet werden. 18 dieser Läsionen waren im Atrium lokalisiert, davon 13 am Trikuspidalklappenanulus und fünf am Mitralklappenanulus. Die restlichen zwölf Läsionen fanden sich im Ventrikelmyokard, wovon drei am ventrikulären Aspekt des Trikuspidalklappen- und neun am ventrikulären Aspekt des Mitralklappenanulus zu finden waren. Die Läsionen imponierten als oval geformte hämorrhagische Areale inmitten von gesundem Myokardgewebe. Die Läsionen waren zudem von einem weißlichen Randsaum umgeben (s. Abbildung 11). 


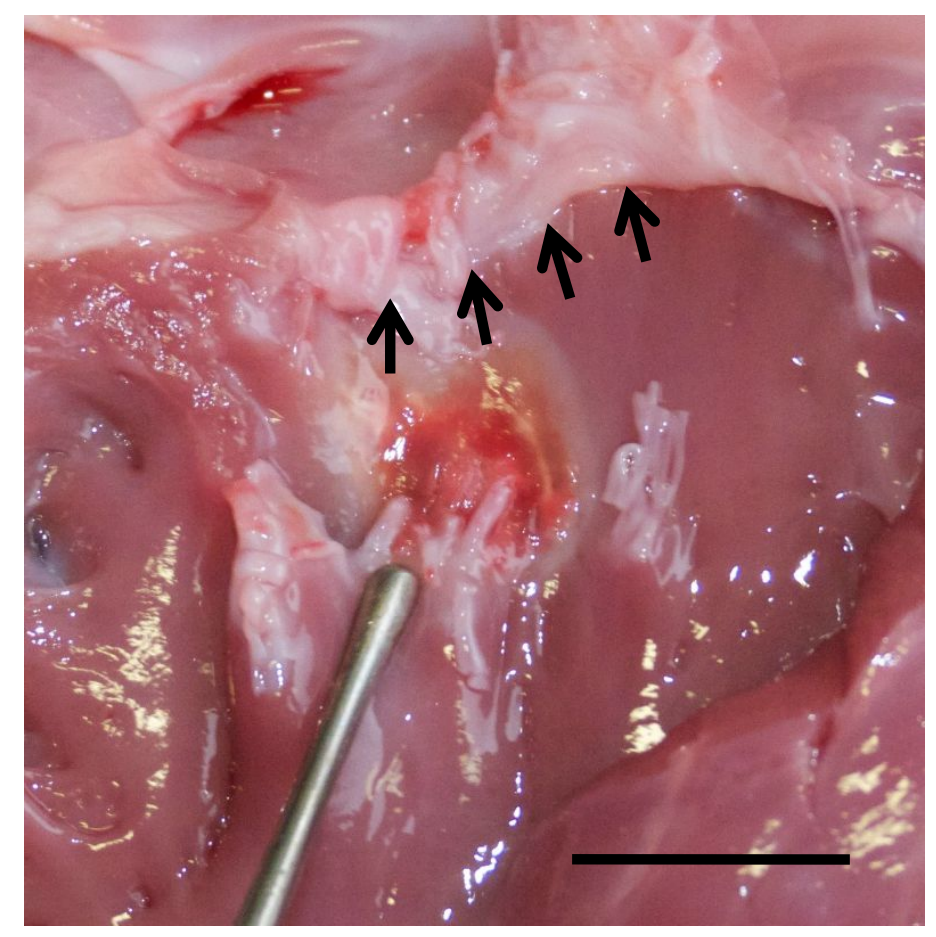

Abbildung 2: Makroskopische Ansicht einer Kryoläsion (Knopfsonde) nach dreifacher Kryoenergieablation am ventrikulären Aspekt der Trikuspidalklappe (Pfeile). Die hämorrhagische Läsion ist durch einen weißen Saum scharf vom vitalen Myokardgewebe abgrenzbar. Skala $=10 \mathrm{~mm}$

\subsection{Mikroskopische Ansicht der Läsionen}

Mikroskopisch imponierten die Läsionen durch eine Auflockerung des Gewebes mit Einblutungen. Der weiße Randsaum, der die Läsionen abgrenzte, zeichnete sich mikroskopisch vor allem durch die Bildung eines reaktiven Ödems sowie eine massive Leukozyteninfiltration aus.

Zur besseren Differenzierung zwischen gesundem und geschädigtem Myokardgewebe wurde mit der Desmin-Färbung eine spezifische immunhistochemische Färbemethode angewandt. Desmin ist ein Intermediärfilament und Bestandteil des Zytoskeletts. In gesundem Myokardgewebe lässt sich Desmin gut anfärben und erscheint bräunlich. Innerhalb der durch dreifache Kryoenergie erzeugten Läsionen war es zur Schädigung des Zytoskellets mit Untergang der Intermediärfilamente gekommen. Das Desmin ließ sich nicht mehr anfärben und das betroffene Areal stellte sich grau dar (s. Abbildung 12). 


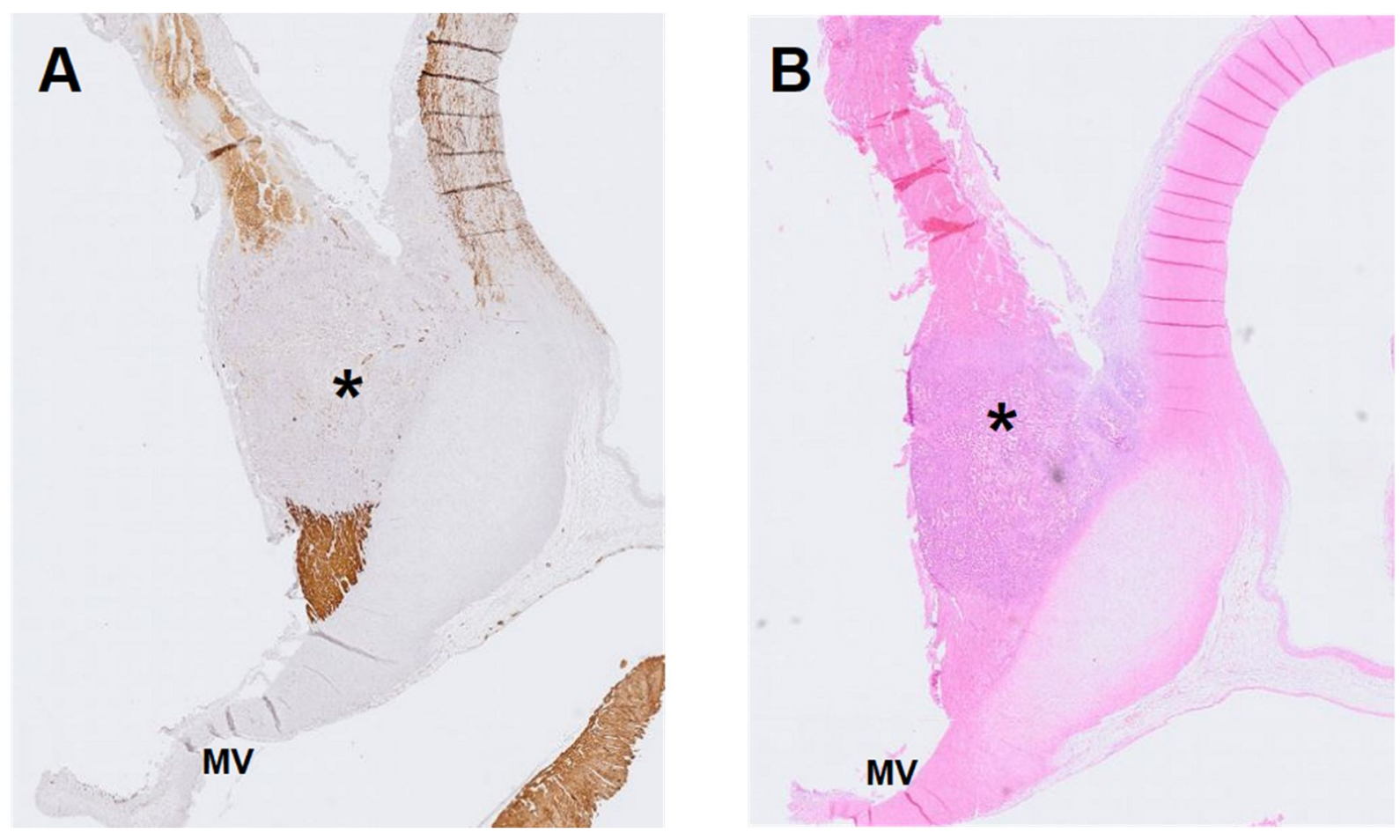

Abbildung 3: Transmurale Kryoläsion (Stern) am atrialen Aspekt der Mitralklappe (MV). A: Desmin-Färbung; B: Hämatoxylin-Eosin-Färbung; Skala $=1 \mathrm{~mm}$.

Auch mikroskopisch ließ sich kein Anhalt für eine Schädigung der Koronararterien dokumentieren. In keinem der Präparate fanden sich Zeichen für pathologische Veränderungen der Gefäßwände oder Lumina. Ebenso wenig fanden sich Thrombenformationen oder Plaques (s. Abbildung 13). 

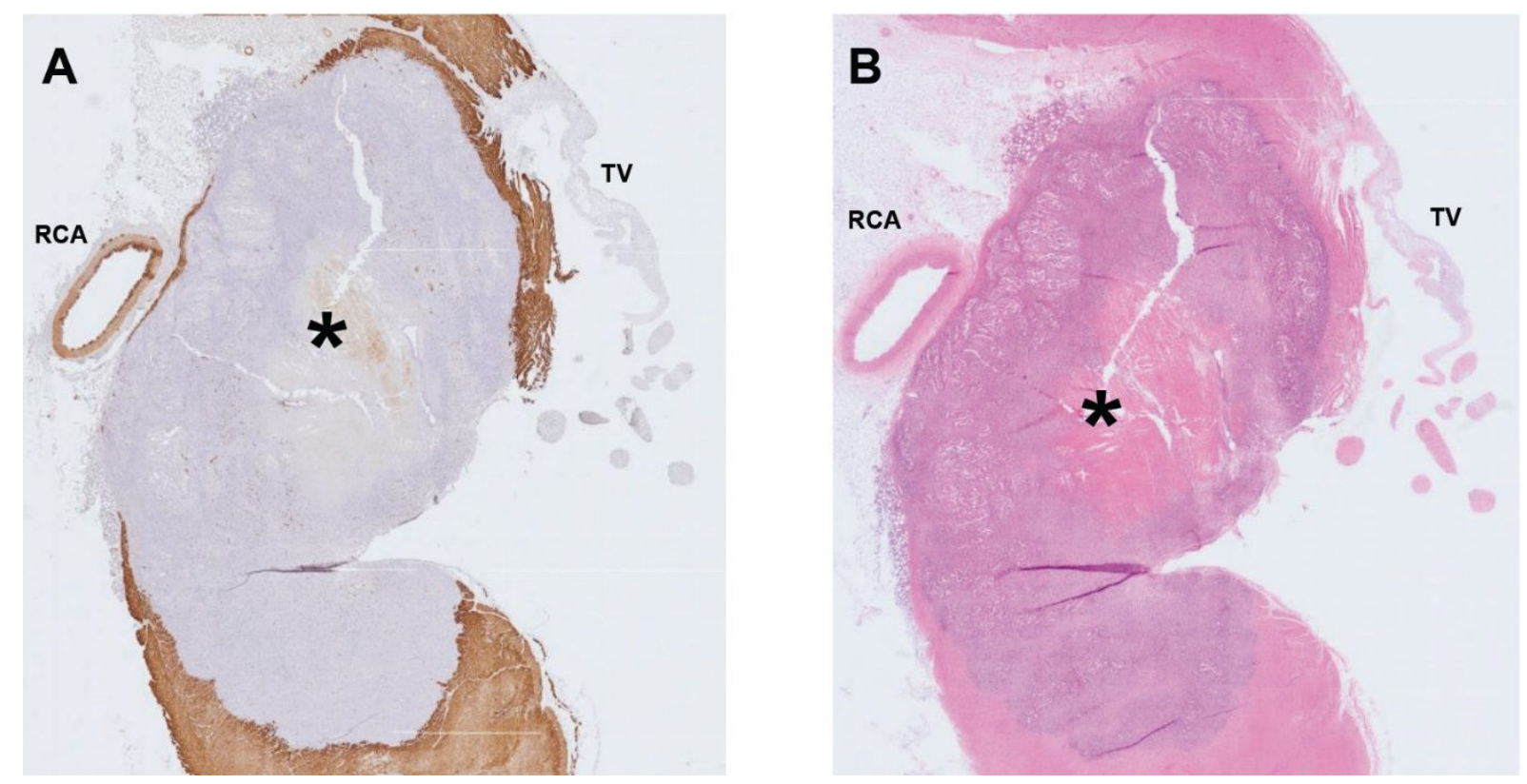

Abbildung 4: Transmurale Kryoläsion (Stern) am ventrikulären Aspekt der Trikuspidalklappe (TV). Am angeschnittenen Teil der rechten Koronararterie (RCA) ist keine pathologische Veränderung nachweisbar. A: Desmin-Färbung; B: Hämatoxylin-Eosin-Färbung; Skala = $1 \mathrm{~mm}$.

\subsection{Planimetrie und Volumetrie}

Im nachfolgenden Teil werden die Läsionsvolumina in Abhängigkeit ihrer Lokalisation beschrieben.

\subsubsection{Atriale Läsionen}

Von den insgesamt 30 identifizierten Kryoläsionen konnten 18 Läsionen (60 \%) auf der atrialen Seite der AV-Klappenringe identifiziert werden. 13 der atrialen Läsionen fanden sich am Trikuspidalklappenring, die verbleibenden fünf atrialen Läsionen konnten am Mitralklappenannulus nachgewiesen werden. Die Einzelwerte für die Volumina der atrialen Läsionen sind in der Tabelle 8 aufgeführt. Die Spannweite der Läsionsvolumina reichte von einem Minimum von $13 \mathrm{~mm}^{3}$ bis zu einem Maximum von 685,4 $\mathrm{mm}^{3}$. Der Mittelwert der atrialen Läsionsvolumina nach dreifacher Kryoablation betrug 190,7 $\pm 167,5 \mathrm{~mm}^{3}$. Alle atrial gelegenen Läsionen wiesen eine transmurale Ausdehnung auf. 
Tabelle 8: Atriale Läsionsvolumina und jeweilige Lokalisation, Trikuspidalklappenring (TK) und Mitralklappenring (MK)

\begin{tabular}{|c|c|c|}
\hline Läsionen & Volumen $\left(\mathrm{mm}^{3}\right)$ & Lokalisation \\
\hline 1 & 121,9 & TK \\
\hline 2 & 425,4 & TK \\
\hline 3 & 137,2 & TK \\
\hline 4 & 241 & TK \\
\hline 5 & 46,5 & TK \\
\hline 6 & 42,3 & TK \\
\hline 7 & 92,7 & TK \\
\hline 8 & 125 & MK \\
\hline 9 & 232,8 & MK \\
\hline 10 & 39,4 & MK \\
\hline 11 & 297,1 & TK \\
\hline 12 & 286,7 & TK \\
\hline 13 & 100,3 & TK \\
\hline 14 & 298,9 & MK \\
\hline 15 & 171,7 & TK \\
\hline 16 & 685,4 & MK \\
\hline 17 & 74,7 & TK \\
\hline 18 & 13 & TK \\
\hline
\end{tabular}

\subsubsection{Ventrikuläre Läsionen}

Von insgesamt 30 Läsionen wurden zwölf Läsionen (40 \%) auf der ventrikulären Seite der AV-Klappenannuli lokalisiert. Die Spannweite der Läsionsvolumina für 
die ventrikulären Läsionen reichte von $44,3 \mathrm{~mm}^{3}$ bis zu einem Maximum von 285,8 $\mathrm{mm}^{3}$. Die Einzelwerte für die Läsionsvolumina sind in Tabelle 9 dargestellt. Der Mittelwert für die ventrikulär gelegenen Läsionen nach dreifacher Kryoablation ergab $184,3 \pm 107,4 \mathrm{~mm}^{3}$. Von den insgesamt zwölf ventrikulär gelegenen Läsionen wiesen nur zwei eine transmurale Ausdehnung auf.

Tabelle 9: Auflistung der ventrikulär gelegenen Kryoläsionen nach Volumen und Lokalisation, Trikuspidalklappenring (TK) und Mitralklappenring (MK)

\begin{tabular}{|c|c|c|}
\hline Läsionen & Volumen $\left.\mathbf{( m m}^{\mathbf{3}}\right)$ & Lokalisation \\
\hline $\mathbf{1}$ & 401,7 & TK \\
\hline $\mathbf{2}$ & 242,8 & MK \\
\hline $\mathbf{3}$ & 161,1 & MK \\
\hline $\mathbf{4}$ & 96 & MK \\
\hline $\mathbf{5}$ & 285,8 & TK \\
\hline $\mathbf{6}$ & 178,6 & MK \\
\hline $\mathbf{7}$ & 44,3 & MK \\
\hline $\mathbf{8}$ & 164,6 & MK \\
\hline $\mathbf{9}$ & 236,7 & MK \\
\hline $\mathbf{1 0}$ & 69,3 & TK \\
\hline $\mathbf{1 1}$ & 268 & MK \\
\hline $\mathbf{1 2}$ & 63,4 & \\
\hline & & \\
\hline & & \\
\hline & & \\
\hline & & \\
\hline & & \\
\hline & & \\
\hline & & \\
\hline & & \\
\hline & & \\
\hline
\end{tabular}

\subsection{Vergleich von atrialen und ventrikulären Läsionsgrößen}

Mit einem Mittelwert der Läsionsvolumina von 184,3 \pm 107,4 mm³ unterschieden sich die ventrikulären Läsionen in ihrer Größe nicht von den atrialen Läsionen $\left(190,7 \pm 167,5 \mathrm{~mm}^{3}, \mathrm{p}=0,24\right)$. 
Die Boxplots in Abbildung 14 veranschaulichen die Verteilung der erhobenen Werte für die Läsionsvolumina in Abhängigkeit zum Ort, an dem die Läsionen generiert wurden.

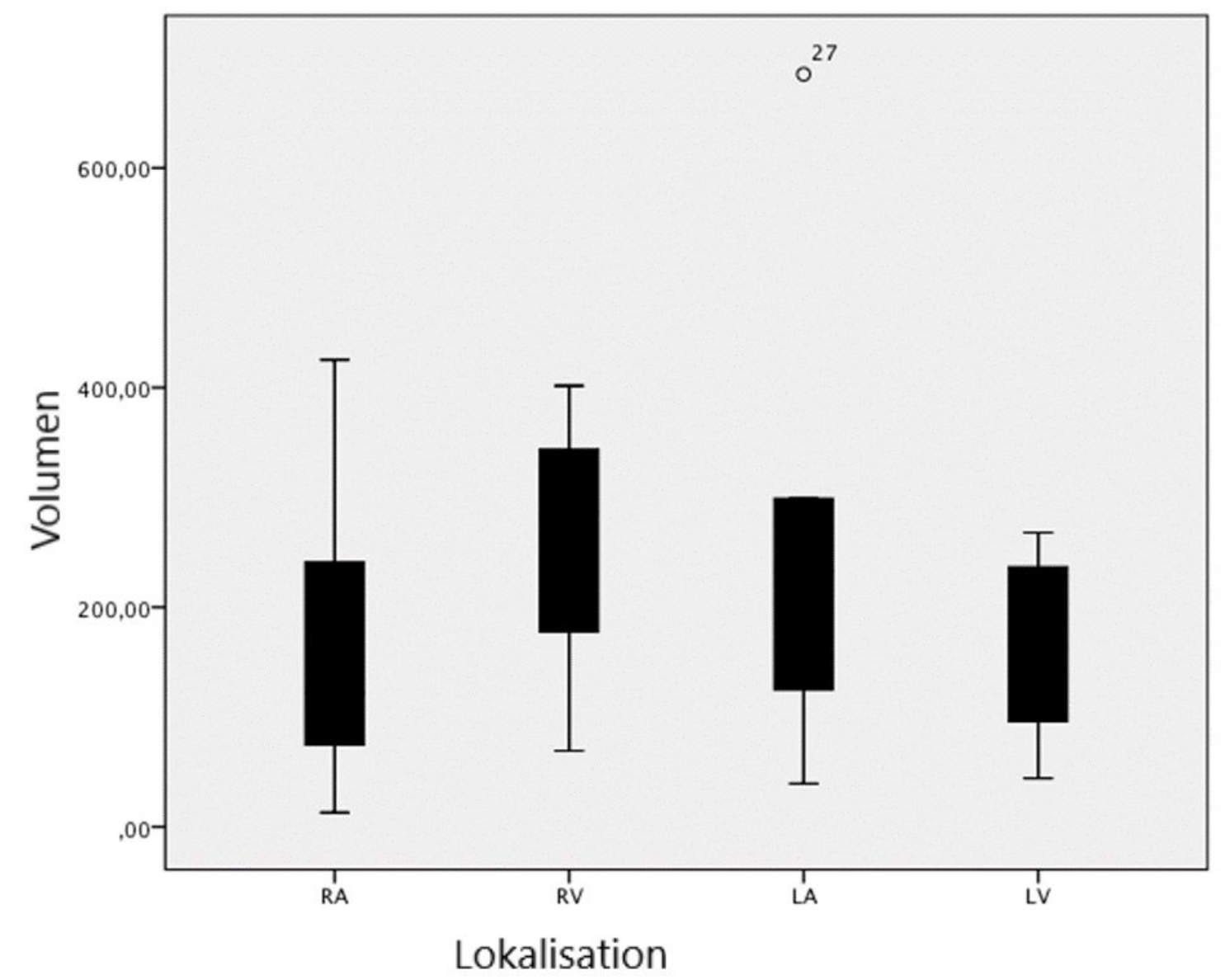

Abbildung 5: Box-Plot der Läsionsvolumina in Abhängigkeit zur Lokalisation. RA = rechtes Atrium, $R V=$ rechter Ventrikel, $L A=$ linkes Atrium, $L V=$ linker Ventrikel

\subsection{Vergleich der Läsionsvolumina nach dreifacher Kryoener- gieapplikation zu Läsionsvolumina nach doppelter Kryoab- lation}

Verglichen mit den Ergebnissen aus Voruntersuchungen, in denen die Auswirkungen von doppelter Kryoenergieablation auf das heranwachsende Myokard untersucht worden waren, fanden sich signifikante Unterschiede der Läsionsvolumina (Schneider et al. 2013). Die Läsionsvolumina waren nach der dreifachen Anwendung von Kryoenergie, verglichen mit den Ergebnissen nach doppelter Kryoenergieanwendung, sowohl im Atrium als auch im Ventrikel größer. Die Ergebnisse sind in der Tabelle 10 dargestellt. 
Tabelle 10: Vergleich der Läsionsvolumina von doppelter (Schneider et al. 2013) und dreifacher Kryoenergieapplikation (Mittelwert $\pm S D$ ) in Abhängigkeit der Lokalisation. $n=$ Anzahl der Läsionen

\begin{tabular}{|c|c|c|c|}
\hline & $\begin{array}{c}\text { Doppelte Kryoenergie } \\
\text { Applikation }\end{array}$ & $\begin{array}{c}\text { Dreifache Kryoenergie } \\
\text { Applikation }\end{array}$ & p-Wert \\
\hline $\begin{array}{l}\text { Atriale Läsio- } \\
\text { nen }\end{array}$ & $\begin{array}{c}109,2 \pm 39,6 \mathrm{~mm}^{3} \\
(\mathrm{n}=16)\end{array}$ & $\begin{array}{c}190,7 \pm 167,5 \mathrm{~mm}^{3} \\
(\mathrm{n}=18)\end{array}$ & 0,004 \\
\hline $\begin{array}{l}\text { Ventrikuläre } \\
\text { Läsionen }\end{array}$ & $\begin{array}{c}150,5 \pm 53,2 \mathrm{~mm}^{3} \\
(\mathrm{n}=15)\end{array}$ & $\begin{array}{c}184,3 \pm 107,4 \mathrm{~mm}^{3} \\
(\mathrm{n}=12)\end{array}$ & 0,034 \\
\hline
\end{tabular}




\section{Diskussion}

In der Therapie von supraventrikulären Tachykardien (SVT) stellt die Kryoenergieablation eine bedeutende Alternative zur Radiofrequenzstrom-Ablation (RFA) dar. Der Vorteil der Kryoenergie, gegenüber der Radiofrequenzenergie, ist das höhere Sicherheitsprofil aufgrund der initialen Reversibilität der Kryoenergieläsionen. Allerdings waren in den meisten zur Kryoenergieablation im Kindesalter publizierten Studien die mittel- und langfristigen Erfolgsraten mit 75 - $87 \%$ niedriger, als nach Radiofrequenzablation, welche eine mittel bis langfristige Erfolgsrate von 90 - 96 \% aufweist (LaPage et al. 2011, Miyazaki et al. 2005a, Kriebel et al. 2005, Bar Cohen et al. 2006). Klinische Studien zeigten, dass eine Verbesserung der Erfolgsrate der Kryoenergieablation durch die Applikation doppelter Kryoenergieläsionen zu erzielen war. Die Erfolgsrate stieg auf 89 - 98 \% (Skanes et al. 2000, Collins et al. 2007, Rodriguez et al. 2002, Drago et al. 2006). Da die Größe der Ablationsläsionen eine wesentliche Determinante für eine erfolgreiche Ablation zu sein scheint, werden unterschiedliche Strategien zur Optimierung der Läsionsgrößen angewandt. Während sich bei der RFA der Katheteranpressdruck an das Gewebe als wichtiger Parameter herausgestellt hat (Kuck et al. 2012) wird bei der Kryoenergieablation durch wiederholte Kryoenergieapplikationen an einer identischen Stelle eine Verbesserung des Ablationserfolges erzielt.

Dieser klinische Befund konnte in einer vorangegangenen tierexperimentellen Studie aus der Klinik für Pädiatrische Kardiologie und Intensivmedizin der Universitätsmedizin Göttingen bestätigt werden. Es konnte gezeigt werden, dass durch die Applikation doppelter Anfrier-Auftau-Zyklen größere Läsionen im Myokard junger Schweine induziert wurden als nach nur einem Anfrier-Auftau-Zyklus (Kriebel et al. 2009, Schneider et al. 2012). Um die Effektivität der Kryoenergieablation weiter zu erhöhen, wurden zuletzt klinisch drei konsekutive Anfrier-Auftau-Zyklen angewandt. Ob ein weiterer Anfrier-Auftau-Zyklus aber tatsächlich zu einer weiteren Größenzunahme der Läsionen führt, mit dem Ziel einer Erhöhung des Ablationserfolgs, ist bislang nicht untersucht worden. Gleichfalls wurde die Auswirkung eines dreifachen Anfrier-Auftau-Zyklus auf sensible anatomische Strukturen des Herzens, wie den AV-Knoten und insbesondere die Koronararterien, nicht systematisch untersucht. 
Da die Größe und Eindringtiefe der Läsionen ein Maß für die Effektivität der Ablation ist (s. o.), sollte untersucht werden, ob bei gleicher Sicherheit eine höhere Effektivität, d. h. eine größere Gewebsläsion, durch drei konsekutive Anfrier-Auftau-Zyklen zu erreichen war.

Die Ergebnisse der vorliegenden Arbeit bestätigen die Annahme, dass bei einer dreifachen Kryoenergieapplikation das Volumen der induzierten Läsionen gegenüber der doppelten Kryoenergieapplikation tatsächlich zunimmt.

Ebenso zeigte sich keine Reduktion des Sicherheitsprofils durch den zusätzlichen Anfrier-Auftau-Zyklus, welches dem der RFA überlegen ist. Allerdings zeichnet sich die RFA in der aktuellen Literatur weiterhin durch eine bessere Effektivität in mittel- und langfristigen Nachbeobachtungen aus (LaPage et al. 2011, Miyazaki et al. 2005b, Kriebel et al. 2005, Bar-Cohen et al. 2006).

Untersuchungen zu den Auswirkungen der Applikation von zweifacher Kryoenergie am heranwachsenden Myokard beschrieben eine gesteigerte Effektivität des unmittelbaren Interventionserfolges sowie die Freiheit von SVT-Rezidiven in mittelfristigen Nachbeobachtungen bei Patienten mit AVNRT (Qureshi et al. 2013, Gist et al. 2009, Drago et al. 2010, Rodriguez et al. 2002). Die klinische Beobachtung, dass durch doppelte Kryoenergieapplikation die Effektivität des Therapieverfahrens erhöht werden konnte, wurde durch den Nachweis größerer Läsionen nach doppelter Kryoenergieapplikation bestätigt (Schneider et al. 2013).

Um die Effektivität der Kryoenergieablation weiter zu steigern, wurde das Konzept der gesteigerten Anzahl an Anfrier-Zyklen aufgegriffen und um einen dritten Zyklus erweitert. Die klinische Anwendung einer dreifachen Kryoenergieapplikation ergab, dass die Effektivität der Ablation mit Kryoenergie durch eine Erhöhung der Anfrier-Auftau-Zyklen zu verbessern ist (Qureshi et al. 2013). Warum unter dreifacher Kryoablation die Effektivität stieg und das Rezidivrisiko sank, wurde in der Publikation von Qureshi et al. allerdings nicht untersucht. Ein weiteres Risiko bei der kathetergeführten Ablation von SVT stellt die potentielle Gefährdung von Koronargefäßen durch die Applikation der zur Ablation des Tachykardiesubstrates notwendigen Energie dar. Deshalb war die Unversehrtheit der Koronararterien nach repetitiver Kryoapplikation ein weiterer wichtiger Endpunkt der Untersuchung.

Die wesentlichen Erkenntnisse der Untersuchung lassen sich wie folgt zusammenfassen: Die Applikation von drei aufeinanderfolgenden Anfrierzyklen für vier 
Minuten, unterbrochen durch 1-minütige Auftauphasen, am heranwachsenden Myokard, führte zu einer signifikanten Zunahme der Läsionsvolumina am Tiermodel. Obwohl die Läsionen größere Ausmaße aufwiesen und so eine höhere Effektivität auf die Substrate tachykarder Herzrhythmusstörungen angenommen werden darf, ließen sich keine Schäden an den Koronargefäßen nachweisen. In der vorangegangenen Studie zu den Auswirkungen von zweifacher Kryoenergieapplikation auf das noch nicht ausgewachsene Myokard waren mittels Koronarangiographie und ICUS keine Veränderungen an den Koronararterien darstellbar. Allerdings stellten sich bei histologischen Untersuchungen der Präparate in $6 \%$ der Läsionen Anomalitäten der Gefäßwände dar. Bei einer Untersuchung zu den Auswirkungen von einfacher Kryoenergieapplikation auf das heranwachsende Myokard waren keine Veränderungen der Koronargefäßwände gefunden worden. In der aktuellen Studie zu dreifacher Kryoapplikation fand sich in den histologischen Untersuchungen ebenfalls kein Anhalt für Veränderungen der Koronararterien, obwohl die Läsionsvolumina signifikant größer waren.

Unter Berücksichtigung der Erkenntnisse, dass die Distanz zwischen Endokard am AV-Klappenannulus und den Koronargefäßen signifikant variiert (Al-Ammouri und Perry 2006), lassen sich die Ergebnisse der Koronargefäßaffektion der Studie, abgesehen von der erhöhten Anzahl an Kryoenergie-Zyklen, auch mit der Lokalisation der gesetzten Läsionen begründen.

Somit erscheint es sinnvoll, vor einer gepalnten Katheterablation (und das nicht nur mit doppelter oder dreifacher Kryoenergieapplikation) am heranwachsenden Myokard, die individuelle Koronaranatomie des Patienten darzustellen, um so enge anatomische Lagebeziehungen zwischen der geplanten Läsionslokalisation und Koronargefäßen zu erkennen.

Im Vergleich zu Untersuchungen zur Läsionsbildung nach Kryoablation wurde in der vorliegenden Untersuchung eine dreidimensionale morphometrische Analyse der Läsionsvolumina durchgeführt. Mittels dieses Vorgehens war es möglich, die Ausmaße der verursachten Läsionen präzise zu ermitteln und mit den Vorergebnissen der Arbeiten der Arbeitsgruppe zu vergleichen.

Es ist bekannt, dass die Größe der Läsionen nach Kryoenergieablation multifaktoriell bedingt ist. Zu den Faktoren zählen unter anderem die Größe der verwendeten Katheterspitze, die Zieltemperatur, mit der abladiert wird, und die Dauer der Ablation (Markovitz et al. 1988). 
Die Ergebnisse der aktuellen Studie deuten eine bevorzugte dreifache Kryoenergie Applikation im klinischen Kontext bei der Ablation von supraventrikulären Substraten am heranwachsenden Myokard an. Allerdings können die Ergebnisse aus tierexperimentellen Studien nicht eins zu eins auf den Menschen übertragen werden. Um die Effektivität und die Sicherheit des untersuchten Ablationsprotokolls mittels modifizierter dreifacher Kryoenergie in der Therapie von supraventrikulären Tachykardien an pädiatrischen Patienten voranzutreiben und zu optimieren, sind weiterführende randomisierte klinische Studien erforderlich. 


\section{Zusammenfassung}

Supraventrikuläre Tachykardien gehören zu den häufigsten symptomatischen Tachyarrhythmien im Kindes-und Jugendalter. Häufig werden diese Tachykardien durch das Vorhandensein akzessorischer Leitungsbahnen oder dualer Leitungseigenschaften des AV-Knotens bedingt. Neben der medikamentösen Therapie hat sich die Katheterablation als Therapiemöglichkeit etabliert. Die Radiofrequenzablation gilt als schnell, effektiv und wenig risikobehaftet. Allerdings kann sie gerade am unreifen Myokard mit signifikanten Gewebeeffekten und Koronarstenosen assoziiert sein. Als sichere und effektive Alternative zur Ablation von arrhythmogenen Substraten gilt die Kryoenergieablation. Zwar gilt die Effektivitätsrate der Kryoablation der der RFA als unterlegen, jedoch zeichnet sie sich durch ein höheres Sicherheitsprofil, vor allem in Bezug auf Koronargefäßaffektionen, aus.

Um die Effektivität der Kryoablation weiter zu steigern, ohne das gewohnt hohe Sicherheitsprofil zu senken, wurde in einer tierexperimentellen Studie die Auswirkung einer modifizierten dreifachen Kryoenergieablation auf die Läsionsgröße und die Koronararterien am heranwachsenden Myokard untersucht. Die Ergebnisse wurden mit denen aus vorangegangenen Untersuchungen zur doppelten Kryoablation verglichen. In sieben Schweineherzen konnten insgesamt 30 Kryoläsionen ausgewertet werden. Von den 30 Kryoläsionen fanden sich 18 im Atrium lokalisiert und zwölf im Ventrikel. Die Läsionsvolumina nach dreifacher Kryoablation zeigten eine signifikante Größenzunahme verglichen mit den Volumina nach doppelter Kryoablation. Trotz Größenzunahme der induzierten Läsionen, konnte bei keinem der Tiere eine Affektion der Koronargefäße oder eine bleibende Herzrhythmusstörung nachgewiesen werden. Somit scheint ein zusätzlicher dritter Anfrier-Auftau-Zyklus das hohe Sicherheitsprofil der Kryoenergieablation nicht zu kompromittieren.

Da die Ergebnisse aus tierexperimentellen Studien nicht eins zu eins auf den Menschen übertragen werden können, ist es erforderlich, weitere randomisierte klinische Studien zur Auswirkung eines modifizierten dreifachen Kryoenergieablationsprotokolls zur Sicherung der Effektivität und der Sicherheit in der Therapie von SVT bei pädiatrischen Patienten durchzuführen. 


\section{Literaturverzeichnis}

Al-Ammouri I, Perry JC (2006): Proximity of coronary arteries to the atrioventricular vaIve annulus in young patients and implications for ablation procedures. Am J Cardiol $\underline{97}, 1752-1755$

Aoyama H, Nakagawa H, Pitha JV, Khammar GS, Chandrasekaran K, Matsudaira K, Yagi T, Yokoyama K, Lazzara R, Jackman WM (2005): Comparison of cryothermia and radiofrequency current in safety and efficacy of catheter ablation within the canine coronary sinus close to the left circumflex coronary artery. $J$ Cardiovasc Electrophysiol $\underline{16}, 1218-1226$

Avitall B, Lafontaine D, Rozmus G, Adoni N, Le KM, Dehnee A, Urbonas A (2004): The safety and efficacy of multiple consecutive cryo lesions in canine pulmonary veins-left atrial junction. Heart Rhythm 1, 203-209

Bar-Cohen Y, Cecchin F, Alexander ME, Berul Cl, Triedman JK, Walsh EP (2006): Cryoablation for accessory pathways located near normal conduction tissues or within the coronary venous system in children and young adults. Heart Rhythm $\underline{3}$, 253-258

Bertram H, Bokenkamp R, Peuster M, Hausdorf G, Paul T (2001): Coronary artery stenosis after radiofrequency catheter ablation of accessory atrioventricular pathways in children with ebstein's malformation. Circulation $\underline{103}, 538-543$.

Blaufox AD, Saul JP (2004): Acute coronary artery stenosis during slow pathway ablation for atrioventricular nodal reentrant tachycardia in a child. J Cardiovasc Electrophysiol $\underline{15}$, 97-100.

Blaufox AD, Paul T, Saul JP (2004): Radiofrequency catheter ablation in small children: relationship of complications to application dose. Pacing Clin Electrophysiol 27, 224-229.

Blomström-Lundqvist C, Scheinman MM, Aliot EM, Alpert JS, Calkins H, Camm AJ, Campbell WB, Haines DE, Kuck KH, Lerman BB (2003): ACC/AHA/ESC guidelines for the management of patients with supraventricular arrhythmias--executive summary. a report of the American college of cardiology/American heart association task force on practice guidelines and the European society of cardiology committee for practice guidelines (writing committee to develop guidelines for the management of patients with supraventricular arrhythmias) developed in collaboration with NASPE-Heart Rhythm Society. J Am Coll Cardiol 42, 1493-531

Bokenkamp R, Wibbelt G, Sturm M, Windhagen-Mahnert B, Bertram H, Hausdorf G, Paul T (2000): Effects of intracardiac radiofrequency current application on coronary artery vessels in young pigs. J Cardiovasc Electrophysiol 11, 565-571.

Borggrefe M, Budde T, Podczeck A, Breithardt G (1987): High frequency alternating current ablation of an accessory pathway in humans. J Am Coll Cardiol $\underline{10}, 576-$ 582.

Brugada J, Blom N, Sarquella-Brugada G, Blomstrom-Lundqvist C, Deanfield J, Janousek J, Abrams D, Bauersfeld U, Brugada R, Drago F, et al. (2013): 
Pharmacological and non-pharmacological therapy for arrhythmias in the pediatric population: EHRA and AEPC-Arrhythmia Working Group joint consensus statement. Europace 15, 1337-1382.

Calkins H, Langberg J, Sousa J, el-Atassi R, Leon A, Kou W, Kalbfleisch S, Morady F (1992): Radiofrequency catheter ablation of accessory atrioventricular connections in 250 patients. Abbreviated therapeutic approach to WolffParkinson-White syndrome. Circulation 85, 1337-1346.

Chatelain P, Zimmermann M, Weber R, Campanini C, Adamec R (1995): Acute coronary occlusion secondary to radiofrequency catheter ablation of a left lateral accessory pathway. Eur Heart J $\underline{16}, 859-861$

Collins KK, Schaffer MS (2011): Use of Cryoablation for Treatment of Tachyarrhythmias in 2010: Survey of Current Practices of Pediatric Electrophysiologists. Pacing Clin Electrophysiol $\underline{34}$, 304-308

Collins KK, Rhee EK, Kirsh JA, Cannon BC, Fish FA, Dubin AM, Van Hare GF (2007): Cryoablation of accessory pathways in the coronary sinus in young patients: a multicenter study from the Pediatric and Congenital Electrophysiology Society's Working Group on Cryoablation. J Cardiovasc Electrophysiol 18, 592-597

Cox JL, Gallagher JJ, Cain ME (1985): Experience with 118 consecutive patients undergoing operation for the Wolff-Parkinson-White syndrome. J Thorac Cardiovasc Surg $\underline{90}, 490-501$

Crick SJ, Sheppard MN, Ho SY, Gebstein L, Anderson RH (1998): Anatomy of the pig heart: comparisons with normal human cardiac structure. J Anat 193,105-119

Czosek RJ, Anderson J, Marino BS, Connor C, Knilans TK (2010): Linear lesion cryoablation for the treatment of atrioventricular nodal re-entry tachycardia in pediatrics and young adults. Pacing Clin Electrophysiol $\underline{33}, 1304-1311$

de Paola AA, Leite LR, Arfelli E (2003): Mechanical reperfusion of acute right coronary artery occlusion after radiofrequency catheter ablation and long-term follow-up angiography. J Invasive Cardiol $\underline{15}, 173-175$

Deisenhofer I, Zrenner B, Yin Y-h, Pitschner H-F, Kuniss M, Grossmann G, Stiller S, Luik A, Veltmann C, Frank J, et al. (2010): Cryoablation Versus Radiofrequency Energy for the Ablation of Atrioventricular Nodal Reentrant Tachycardia (the CYRANO Study): Results From a Large Multicenter Prospective Randomized Trial. Circulation 122, 2239-2245

Drago F, De Santis A, Grutter G, Silvetti MS (2005): Transvenous cryothermal catheter ablation of re-entry circuit located near the atrioventricular junction in pediatric patients: efficacy, safety, and midterm follow-up. J Am Coll Cardiol $\underline{45}, 1096-$ 1103

Drago F, Silvetti M, De Santis A, Grutter G, Andrew P (2006): Lengthier cryoablation and a bonus cryoapplication is associated with improved efficacy for cryothermal catheter ablation of supraventricular tachycardias in children. J Interv Card Electrophysiol 16, 191-198 
Drago F, Russo MS, Silvetti MS, DE Santis A, lodice F, Naso Onofrio MT (2010): Cryoablation of typical atrioventricular nodal reentrant tachycardia in children: six years' experience and follow-up in a single center. Pacing Clin Electrophysiol $\underline{33}$, $475-481$

Dubuc M, Roy D, Thibault B, Ducharme A, Tardif JC, Villemaire C, Leung TK, Talajic M (1999): Transvenous catheter ice mapping and cryoablation of the atrioventricular node in dogs. Pacing Clin Electrophysiol 22, 1488-1498

Gaita F, Haissaguerre M, Giustetto C, Grossi S, Caruzzo E, Bianchi F, Richiardi E, Riccardi R, Hocini M, Jais P (2003): Safety and efficacy of cryoablation of accessory pathways adjacent to the normal conduction system. J Cardiovasc Electrophysiol 14, 825-829

Gillette PC, Garson A Jr, Porter CJ, Ott D, McVey P, Zinner A, Blair H. (1983): Junctional automatic ectopic tachycardia: new proposed treatment by transcatheter His bundle ablation. Am Heart J $\underline{106}, 619-623$

Gist KM, Bockoven JR, Lane J, Smith G, Clark JM (2009): Acute success of cryoablation of left-sided accessory pathways: a single institution study. J Cardiovasc Electrophysiol 20, 637-642

Holman WL, Ikeshita M, Ungerleider RM, Smith PK, Ideker RE, Cox JL (1983): Cryosurgery for cardiac arrhythmias: acute and chronic effects on coronary arteries. Am J Cardiol $\underline{51}, 149-155$

Khanal S, Ribeiro PA, Platt M, Kuhn MA (1999): Right coronary artery occlusion as a complication of accessory pathway ablation in a 12-year-old treated with stenting. Catheter Cardiovasc Interv $\underline{46}, 59-61$

Kimman GP, Theuns DA, Szili-Torok T, Scholten MF, Res JC, Jordaens LJ (2004): CRAVT: a prospective, randomized study comparing transvenous cryothermal and radiofrequency ablation in atrioventricular nodal re-entrant tachycardia. Eur Heart J $\underline{25}, 2232-2237$

Kirsh JA, Gross GJ, O'Connor S, Hamilton RM (2005): Transcatheter cryoablation of tachyarrhythmias in children: initial experience from an international registry. $J$ Am Coll Cardiol $\underline{45}, 133-136$

Koch J, Lücke C, Gebauer R, Hindricks G, Arya A. (2016): Accessory pathway ablation in childhood forming the substrate for ventricular tachycardia in adulthood. Eur Heart J $\underline{37}, 609$

Krause U, Abreu da Cunha FD, Backhoff D, Jacobshagen C, Klehs S, Schneider HE, Paul T (2017): Effects of Triple Cryoenergy Application on Lesion Formation and Coronary Arteries in the Developing Myocardium. Pediatr Cardiol $\underline{38}$, 663-668

Kriebel T, Broistedt C, Kroll M, Sigler M, Paul T (2005): Efficacy and safety of cryoenergy in the ablation of atrioventricular reentrant tachycardia substrates in children and adolescents. J Cardiovasc Electrophysiol $\underline{16}$, 960-966 
Kriebel T, Schneider H, Paul T (2007a): Entstehungsweise und Beeinflussung von tachykarden Herzrhythmusstörungen im Kindesalter. Somnologie 11, 9-15

Kriebel T, Saul JP, Schneider H, Sigler M, Paul T (2007b): Noncontact mapping and radiofrequency catheter ablation of fast and hemodynamically unstable ventricular tachycardia after surgical repair of tetralogy of Fallot. J Am Coll Cardiol 무, 2162-2168

Kriebel T, Hermann HP, Schneider H, Kroll M, Selle J, Overwaul A, Sigler M, Paul T (2009): Cryoablation at growing myocardium: no evidence of coronary artery obstruction or intimal plaque formation early and late after energy application Pacing Clin Electrophysiol $\underline{32}, 1197-1202$

Kuck KH, Reddy VY, Schmidt B, Natale A, Neuzil P, Saoudi N, Kautzner J, Herrera C, Hindricks G, Jaïs P, Nakagawa H, Lambert H, Shah DC (2012): A novel radiofrequency ablation catheter using contact force sensing: Toccata study. Heart Rhythm $\underline{9}, 18-23$

Kugler JD, Danford DA, Deal BJ, Gillette PC, Perry JC, Silka MJ, Van Hare GF, Walsh EP (1994): Radiofrequency catheter ablation for tachyarrhythmias in children and adolescents. The Pediatric Electrophysiology Society. N Engl J Med $\underline{330}, 1481$ 1487

Kugler JD, Danford DA, Houston K, Felix G (1997): Radiofrequency catheter ablation for paroxysmal supraventricular tachycardia in children and adolescents without structural heart disease. Pediatric EP Society, Radiofrequency Catheter Ablation Registry. Am J Cardiol 으, 1438-1443

Kugler JD, Danford DA, Houston KA, Felix G (2002): Pediatric Radiofrequency Catheter Ablation Registry Success, Fluoroscopy Time, and Complication Rate for Supraventricular Tachycardia: Comparison of Early and Recent Eras. J Cardiovasc Electrophysiol 13, 336-341

Lanzotti ME, De Ponti R, Tritto M, Spadacini G, Salerno-Uriarte JA (2002): Successful treatment of anteroseptal accessory pathways by transvenous cryomapping and cryoablation. Ital Heart J $\underline{3}, 128-132$

LaPage MJ, Saul JP, Reed JH (2010): Long-term outcomes for cryoablation of pediatric patients with atrioventricular nodal reentrant tachycardia. Am J Cardiol 105, 1118-1121

LaPage MJ, Reed JH, Collins KK, Law IH, Pilcher TA, Tanel RE, Anderson CC, Young ML, Emmel M, Paul T, Blaufox AD, Arora G, Saul JP (2011): Safety and results of cryoablation in patients $<5$ years old and/or $<15$ kilograms. Am J Cardiol $\underline{108}$, 565-571

Lavergne T, Le Heuzey JY, Bruneval P, Guize L, Boutjdir M, Von Euw D, Peronneau P (1986): Effects of physical parameters of fulguration on electrophysiological and anatomical properties of canine myocardium. Pacing Clin Electrophysiol $\underline{9}, 1367-$ 1375 
Lowe MD, Meara M, Mason J, Grace AA, Murgatroyd FD (2003): Catheter cryoablation of supraventricular arrhythmias: a painless alternative to radiofrequency energy. Pacing Clin Electrophysiol 26, 500-503

Lustgarten DL, Keane D, Ruskin J (1999): Cryothermal ablation: Mechanism of tissue injury and current experience in the treatment of tachyarrhythmias. Progress in Cardiovascular Diseases $\underline{41}$, 481-498

Manusama R, Timmermans C, Philippens S, Crijns HJ, Ayers GM, Rodriguez LM (2004): Single cryothermia applications of less than five minutes produce permanent cavotricuspid isthmus block in humans. Heart Rhythm 1, 594-599

Markovitz LJ, Frame LH, Josephson ME, Hargrove WC 3rd (1988): Cardiac cryolesions: factors affecting their size and a means of monitoring their formation. Ann Thorac Surg $\underline{46}, 531-535$

Mazur P (1970): Cryobiology: The Freezing of Biological Systems. Science 168, 939949

Miyazaki A, Blaufox AD, Fairbrother DL, Saul JP (2005a): Cryo-ablation for septal tachycardia substrates in pediatric patients: mid-term results. J Am Coll Cardiol $\underline{45}, 581-588$

Miyazaki A, Blaufox AD, Fairbrother DL, Saul JP (2005b): Prolongation of the fast pathway effective refractory period during cryoablation in children: a marker of slow pathway modification. Heart Rhythm 2, 1179-1185

Morady F (2004): Catheter Ablation of Supraventricular Arrhythmias. J Cardiovasc Electrophysiol $\underline{15}, 124-139$

Paul T, Bokenkamp R, Mahnert B, Trappe HJ (1997): Coronary artery involvement early and late after radiofrequency current application in young pigs. Am Heart $\mathrm{J}$ $\underline{133}, 436-440$

Paul T, Gebauer R, Kriebel T, Schneider H, Janousek J (2018): Pädiatrische Kradiologie: Tachykarde Herzrhythmusstörungen. S2k-Leitlinie der Deutschen Gesellschaft für Pädiatrische Kardiologie. https://www.awmf.org/uploads/tx_szleitlinien/023-022I_S2k_Tachykarde_Herzrhythmusstoerungen-Kinder-Jugendlichejunge-Erwachsene_2019-04.pdf; abgerufen am 16.09.2019

Pons M, Beck L, Leclercq F, Ferriere M, Albat B, Davy JM (1997): Chronic left main coronary artery occlusion: a complication of radiofrequency ablation of idiopathic left ventricular tachycardia. Pacing Clin Electrophysiol 20, 1874-1876

Qureshi MY, Ratnasamy C, Sokoloski M, Young ML (2013): Low Recurrence rate in treating atrioventricular nodal reentrant tachycardia with triple freeze-thaw cycles. Pacing Clin Electrophysiol $\underline{36}, 279-285$

Riccardi R, Gaita F, Caponi D, Grossi S, Scaglione M, Caruzzo E, Di Donna P, Pistis G, Richiardi E, Giustetto C, Bocchiardo M (2003): Percutaneous catheter cryothermal ablation of atrioventricular nodal reentrant tachycardia: efficacy and safety of a new ablation technique. Ital Heart $\mathrm{J} \underline{4}, 35-43$ 
Rodriguez LM, Geller JC, Tse HF, Timmermans C, Reek S, Lee KL, Ayers GM, Lau CP, Klein HU, Crijns HZ (2002): Acute results of transvenous cryoablation of supraventricular tachycardia (atrial fibrillation, atrial flutter, Wolff-Parkinson-White syndrome, atrioventricular nodal reentry tachycardia). J Cardiovasc Electrophysiol $\underline{13}$, 1082-1089

Ruskin JN (1991): Catheter ablation for supraventricular tachycardia. N Engl J Med $\underline{324}, 1660-1662$

Saul JP, Hulse JE, Papagiannis J, Van Praagh R Walsh EP (1994): Late enlargement of radiofrequency lesions in infant lambs. Implications for ablation procedures in small children. Circulation $\underline{90}$, 492-499

Saul JP, Kanter RJ, Writing C, Abrams D, Asirvatham S, Bar-Cohen Y, Blaufox AD, Cannon B, Clark J, Dick M (2016): PACES/HRS expert consensus statement on the use of catheter ablation in children and patients with congenital heart disease: Developed in partnership with the Pediatric and Congenital Electrophysiology Society (PACES) and the Heart Rhythm Society (HRS). Endorsed by the governing bodies of PACES, HRS, the American Academy of Pediatrics (AAP), and the American Heart Association (AHA). Heart Rhythm 13, 251-289

Schaffer MS, Gow RM, Moak JP, Saul JP (2000): Mortality following radiofrequency catheter ablation (from the Pediatric Radiofrequency Ablation Registry). Participating members of the Pediatric Electrophysiology Society. Am J Cardiol $\underline{86}, 639-643$

Scheinman MM, Huang S (2000): The 1998 NASPE prospective catheter ablation registry. Pacing Clin Electrophysiol $\underline{23}$, 1020-1028

Schneider HE, Kriebel T, Gravenhorst VD, Paul T (2009): Incidence of coronary artery injury immediately after catheter ablation for supraventricular tachycardias in infants and children. Heart Rhythm $\underline{6}, 461-467$

Schneider HE, Kriebel T, Schillinger W, Schill M, Jakobi J, Paul T (2012): Double cryoenergy application (freeze-thaw-freeze) at growing myocardium: lesion volume and effects on coronary arteries early after energy application. Implications for efficacy and safety in pediatric patients. J Cardiovasc Electrophysiol 24, 701-707

Schneider HE, Kriebel T, Groene N, Schillinger W, Stahl M, Schill M, Paul T: Double cryoenergy application (freeze-thaw-freeze) at growing myocardium: histologic evaluation of ablation lesion dimension and coronary artery affection late after

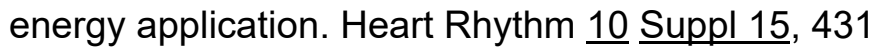

Sealy WC, Gallagher JJ, Wallace AG (1976): The surgical treatment of Wolff-Parkinson-White Syndrome: evolution of improved methods for identification and interruption of the Kent Bundle. Ann Thorac Surg 22, 443-457

Sealy WC, Gallagher JJ (1981): Surgical treatment of left free wall accessory pathways of atrioventricular conduction of the Kent type. J Thorac Cardiovasc Surg 81, 698-706 
Silver ES, Silva JN, Ceresnak SR, Chiesa NA, Rhee EK, Dubin AM, Avasarala K, Van Hare GF, Collins KK (2010): Cryoablation with an 8-mm tip catheter for pediatric atrioventricular nodal reentrant tachycardia is safe and efficacious with a low incidence of recurrence. Pacing Clin Electrophysiol $\underline{33}, 681-686$

Skanes AC, Dubuc M, Klein GJ, Thibault B, Krahn AD, Yee R, Roy D, Guerra P, Talajic $M$ (2000): Cryothermal ablation of the slow pathway for the elimination of atrioventricular nodal reentrant tachycardia. Circulation 102, 2856-2860

Skanes AC, Klein G, Krahn A, Yee R (2004): Cryoablation: potentials and pitfalls. J Cardiovasc Electrophysiol 15, 28-34

Strobel GG, Trehan S, Compton S, Judd VE, Day RW, Etheridge SP (2001): Successful Pediatric Stenting of a Nonthrombotic Coronary Occlusion as a Complication of Radiofrequency Catheter Ablation. Pacing Clin Electrophysiol $\underline{24}$, 1026-1028

Van Hare GF, Velvis H, Langberg JJ (1990): Successful transcatheter ablation of congenital junctional ectopic tachycardia in a ten-month-old infant using radiofrequency energy. Pacing Clin Electrophysiol $\underline{13}$, 730-735

Vedel J, Frank R, Fontaine G, Fournial JF, Grosgogeat Y (1979): Permanent intra-hisian atrioventricular block induced during right intraventricular exploration. Arch Mal Coeur Vaiss $\underline{72}, 107-112$

Weber H, Schmitz L (1983): Catheter technique for closed-chest ablation of an accessory atrioventricular pathway. N Engl J Med $\underline{308}, 653-654$

Wittkampf FH, Wever EF, Derksen R, Wilde AA, Ramanna H, Hauer RN, Robles de Medina EO (1999): LocaLisa: new technique for real-time 3-dimensional localization of regular intracardiac electrodes. Circulation 99, 1312-1317 


\section{Internetquellenverzeichnis}

https://en.ecgpedia.org/index.php?title=File:OCT_ACT.svg; zuletzt abgerufen am 16.09.2019

https://www.ecgguru.com/sites/default/files/ecg-heart-art/AP\%20vs\%20Sinus\%20C\%20Watford_0.jpg; zuletzt abgerufen am 16.09.2019

https://en.ecgpedia.org/index.php?title=File:De-Re_entry.png; zuletzt abgerufen am 16.09.2019

https://de.wikipedia.org/wiki/Kryoablation\#/media/Datei:Cryoablation.jpg; zuletzt abgerufen am 16.09.2019 